\title{
Energy issues in microwave food processing: a review of developments and the enabling potentials of solid-state power delivery
}

\author{
JC Atuonwu, SA Tassou
}

\begin{abstract}
The enormous magnitude and variety of microwave applications in household, commercial and industrial food processing creates a strong motivation for improving the energy efficiency and hence, sustainability of the process. This review critically assesses key energy issues associated with microwave food processing, focusing on previous energy performance studies, energy performance metrics, standards and regulations. Factors affecting energy-efficiency are categorised into source, load and source-load matching factors. This highlights the need for highly-flexible and controllable power sources capable of receiving real-time feedback on load properties, and effecting rapid control actions to minimise reflections, heating non-uniformities and other imperfections that lead to energy losses. A case is made for the use of solidstate amplifiers as alternatives to conventional power sources, magnetrons. By a fullscale techno-economic analysis, it is shown that the use of solid-state amplifiers as replacements to magnetrons is promising, not only from an energy and overall technical perspective, but also in terms of economics.
\end{abstract}

Keywords: Microwave food processing; energy; sustainability; solid-state power sources; emerging process technologies; economics

\section{Introduction}

The significant rise in consumer demand for minimally-processed, microbiologicallysafe and chemical additive-free foods, demands continuous innovation in food processing. As traditional thermal processing systems are limited by low heat transfer rates, and hot wall with cold centre effects, volumetric heating methods are desirable for delivering safe foods of high nutritional and sensory quality. Microwave heating is a promising technology in this regard, finding increasing application in a wide variety of domestic, commercial and industrial food processing operations, globally. As at 2014, about $92 \%$ of UK households have microwave ovens, mainly for food (ONSUK, 2017, - Fig. 1). A market penetration rate of 101\% (some households have multiple microwave ovens), was reported in the UK in 2016 (Euromonitor International, 2017). Over 30 million microwave ovens are sold annually world over (Microwave Technologies Association, 2017), with about 11.33 million unit shipments within the United States in 2016 (ApplianceMagazine.com, 2017). Other reports (HEECS, 2010) suggest that in Europe only, domestic microwave ovens consume about 12.4 TWh of energy per year, with relatively poor overall efficiency (electricity-to-heat) of $50 \%$ or less. A screening life cycle analysis LCA on microwave ovens by Samsung Electronics Inc. (Kim, et al., 1996) concluded that the energy consumption in the use-phase has the highest sustainability impact, compared to the raw material extraction, production, distribution and after-use phases. In addition to all these, industrial and commercial 


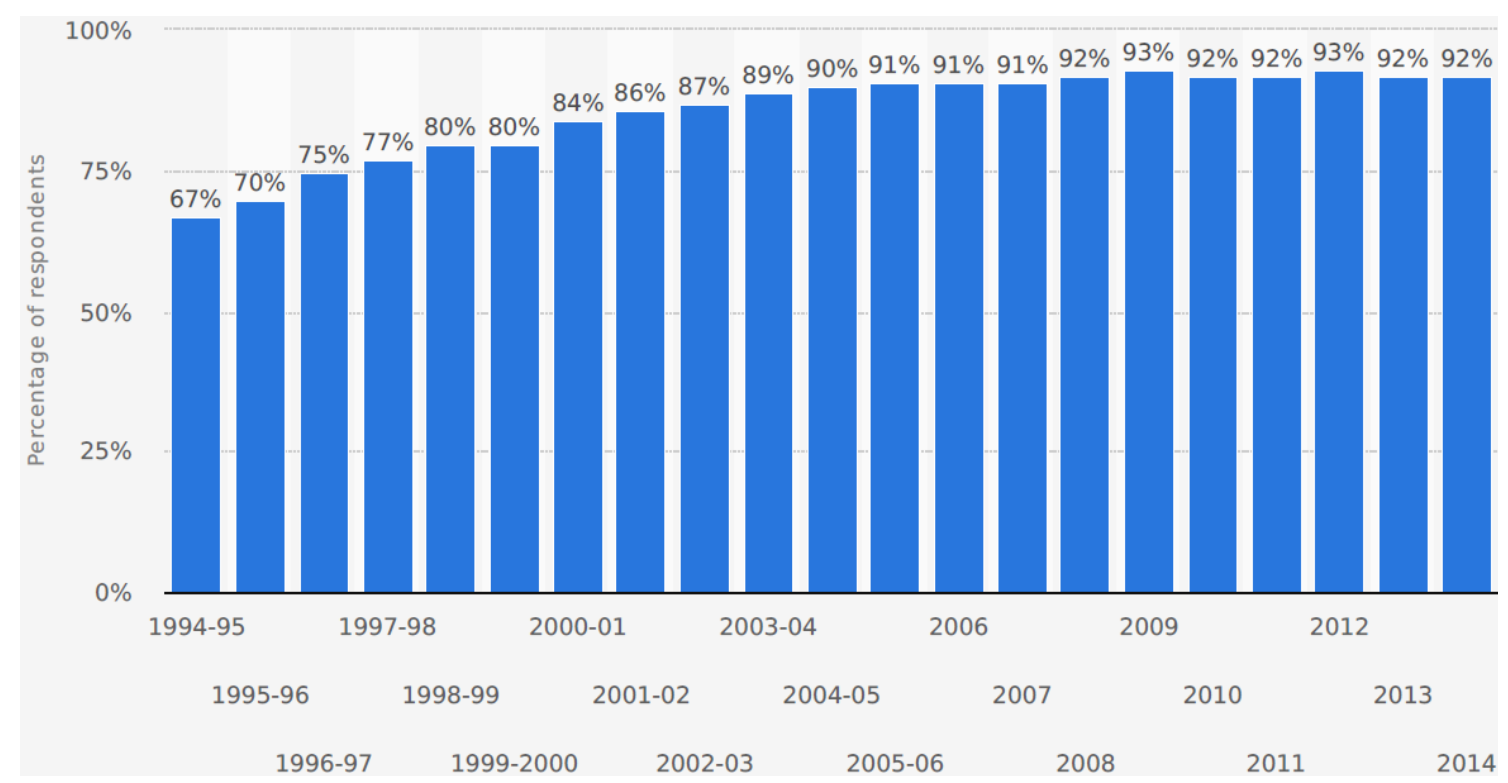

Fig. 1. Percentage of households with microwave ovens in the United Kingdom from 1994-2004

applications of microwave heating in food processing, is on the rise, for instance in tempering of frozen meat and poultry products; precooking of bacon for foodservice and cooking of sausages (Ahmed and Ramaswamy, 2007). Other applications include food drying; baking of bread, biscuit, and confectionery; thawing of frozen products; blanching of vegetables; heating, sterilisation and pasteurisation of fast food, cooked meals, and other foods. Given the widespread end-use, any energy savings in microwave food processing would have a large overall sustainability impact.

Although faster and more volumetric than conventional heating, microwave heating suffers from non-uniform volumetric temperature distribution due to the uneven distribution of dielectric and thermal properties across most foods, as well as microwave standing wave and finite penetration depth phenomena. The result is the formation of cold spots which define the limits of food microbial safety, and hot spots, associated with the degradation of vital product sensory and nutritional quality attributes. One significant contributor to energy losses is the use of higher than optimal temperature-time combinations to guarantee microbial safety at the cold spots. The existence of these non-uniformities can be translated to inefficient energy distribution per unit volume of the product. A perfect energy distribution would be one that results in uniform heat load per-unit volume across the entire product. A more uniform distribution would enable the process to be steered to operate within tight constraints which would promote energy efficiency. Improving temperature uniformity is thus an important issue for energy efficiency. This is a challenging problem, relevant to a wide variety of solid/multiphase foods such as fresh and frozen meat and fish products, fruits and vegetables, grains, etc. of various geometries, and to a lesser extent, liquid foods like juices and dairy. Vadivambal and Jayas (2010) gives a historical review of experimental and modelling studies on the problem of non-uniform microwave heating and proposed ameliorating solutions. Similar reviews, for the specific case of microwave drying were conducted by Li et al. (2011). 
More recently, reviews on diverse areas of microwave food processing have appeared in the literature. Salazar-Gonzalez et al. (2012) reviewed the microwave heating of various fluid foods and their dielectric properties. Chandrasekaran et al. (2013) reviewed microwave mechanisms, food dielectric properties and their measurement methods as well as various stand-alone microwave food processes like cooking, blanching, pasteurisation, sterilisation, drying and drying-based combination operations such as microwave-assisted freeze drying. Guo et al. (2017), performed similar studies with more recent examples. Ekezie et al. (2017) focussed on combination microwave operations such as microwave-assisted ultrasonic, infrared and ohmic operations, concluding that most of these technologies are still at experimental phases, with hardly any report on pilot or industrial-scale facilities. Datta and Rakesh (2013) detailed the practical principles of microwave combination heating. Tang et al. (2015) examined the basic microwave properties relevant to heating uniformity, presenting a historical overview on the development of microwave-assisted thermal sterilisation and pasteurisation systems in research laboratories and used in food plants. Emphasis was on bridging the gap between academic research and industrial implementation. Atuonwu and Tassou (2017) reviewed microwave food processing quality assurance issues such as process lethality, product degradation mechanisms, non-uniform heating and volumetric temperature sensing; showing the energy cost implications of non-uniform heating and proposing the use of solid-state devices for improved quality. The purpose of the current article is to critically analyse the issue of energy consumption in domestic and industrial microwave food processing systems, bringing together a representative number of related experimental and modelling studies. Areas of focus include energy consumption analysis of various microwave food processes; energy performance indices and metrics; energy standardisation and labelling issues; factors affecting energy efficiency and finally, the possibilities (and challenges) from an energy perspective of using solid-state devices instead of the currently-deployed magnetrons for microwave power delivery.

\section{Principles, features and applications}

\subsection{Background}

Microwaves are electromagnetic waves in the frequency range of $300 \mathrm{MHz}$ to $300 \mathrm{GHz}$. Originally deployed in telecommunication systems, the idea of using microwaves for food processing was accidentally discovered in 1945 by Percy Spencer of the Raytheon Company on observing the melting of candle bars in his pocket by radar waves. Subsequent experiments showed that microwaves could raise the internal temperature of many foods far more rapidly than conventional ovens. The first patent, describing an industrial conveyor belt microwave system, was issued in 1952 (Spencer, 1952), however its first application did not start until about 10 years later (Shaheen, et al., 2012). Nowadays, microwave heating systems are usually restricted to certain frequencies, within the so-called industrial, scientific, and medical (ISM) band. The most-commonly used frequencies are $896 \mathrm{MHz}$ (in the UK) and $915 \mathrm{MHz}$ (in the Americas and China), for industrial applications; and $2450 \mathrm{MHz}$, for commercial and domestic uses in most countries. $5800 \mathrm{MHz}$ is available for exploratory purposes. 
Microwave heaters are characterised by non-requirement of ancillary equipment, like boilers and their associated infrastructure; absence of hot surfaces, burn-on, fouling and flavour taints in processed foods; thus reducing cleaning times and frequencies, chemical use in cleaning operations and the volume of waste water.

\subsection{Constructional features and operational principles}

Fig. 2 shows a basic schematic of a generalised microwave heating system (with provision for hybrid or combination operation). It consists essentially of a power supply system (transformers and power conditioning electronic circuits), microwave power source, usually a magnetron with its radiating antenna, a waveguide, and an applicator/heating cavity. The mains voltage is stepped up by initially by the mains transformer. It is increased further by a voltage multiplier electronic circuit, which also acts as a rectifier/filter, converting the transformer output to a pulsed DC voltage. The high DC voltage is then applied between the cathode and anode of the magnetron. The filament transformer on the other hand, steps down the mains voltage to a few Volts AC, which is applied to a filament, concentric with, and housed within the cathode of the magnetron. The magnetron, which is a vacuum tube, is the heart of the microwave system as it generates the microwave energy. As shown in Fig. 3 (I), it consists essentially of the cathode, anode and a static magnetic field source (permanent magnet). The cathode, is made up of a hollow cylinder of emissive material surrounding the heating filament and located in the centre of the anode structure, while the anode is made of a highly conductive material, machined to have cavities. Different anode cavity configurations exist as shown in Fig. 2(II): the slot-type (a), vane-type (b), rising-sun-type (c) and the hole-and-slot-type (d). Of these, the vane type is commonly used for microwave power heating applications. The parallel (or near-parallel) tip sides form a capacitor, while the continuous rear walls form a oneturn inductor coil. Hence, each cavity can be represented by a parallel inductancecapacitance (LC) tuned circuit (Fig. 3(III)).

Initially, the cathode, by means of the filament voltage, is heated up indirectly via the filament to emit electrons by a process known as thermionic emission. The electrons are then accelerated towards the anode by the high-power supply DC voltage. The magnetic field due to the permanent magnet is at right angles to the electronaccelerating electric field, and interacts with it, producing a Lorentz force on the electrons. This force deflects the electrons from their normal straight paths, so they follow circular or cycloidal paths. This leads to bunching of the electrons in a pattern that resembles the spokes in a spinning wheel (Fig. 3(I)), commonly referred to as the space-charge wheel. As this electron cloud approaches a cavity gap, they give off a small part of energy to the cavities. This energy tends to repel negative charges present in the nearby anode segment, causing them to flow round the inductive loop. The inductive current establishes a magnetic field which subsequently establishes a charging current to the capacitive part. A capacitive charge-discharge cycle and corresponding inductive magnetic field build up-collapse cycle is set up, which establishes alternating electric and magnetic fields in that segment of the anode. 


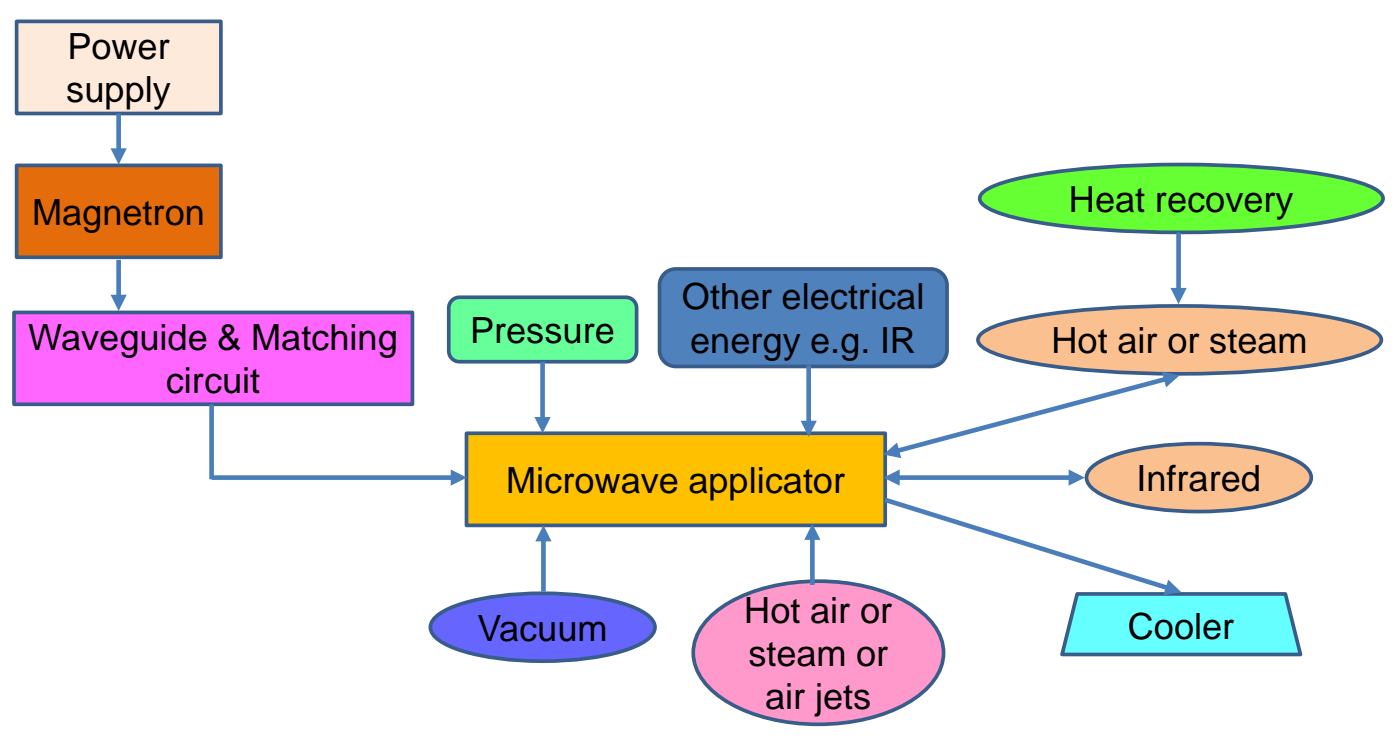

Fig. 2. Schematic of basic generalised microwave heating system (adapted from Metaxas, 1991)

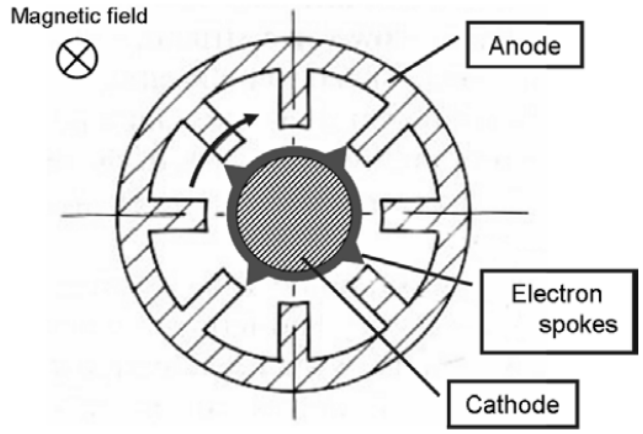

(l)

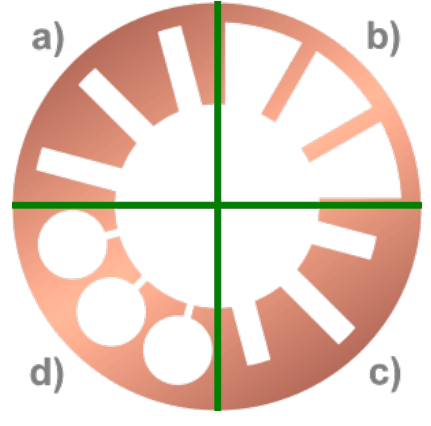

(II)

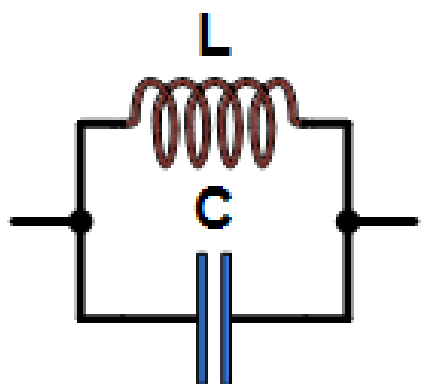

(III)

Fig. 3. (I) Structure of the magnetron - Carter, 2013 (II) different configurations of an anode cavity Wolff, 2017 (III), equivalent circuit of each cavity

Similar processes occur in all the segments and corresponding LC circuits. By the right-hand screw rule, the alternating magnetic field is perpendicular to the alternating electric field. This is the condition for electromagnetic wave generation in the third mutually-perpendicular direction. The microwaves thus generated are tapped via an output coupling loop, and channelled via the waveguide to the heating cavity, through the applicator, for onward interactions with the processed product. In general, part of the microwave energy is absorbed by the product, the magnitude of which depends on its dielectric properties. The rest is reflected towards the magnetron (usually trapped by circulators). A microwave processing system may be operated in batch or continuous mode. In batch mode, the product is placed in the heating cavity and processed until the processing time elapses. The heating cavity is usually equipped with a turntable to promote uniformity of heating. In continuous mode, the product is fed continuously into the heating cavity using either a conveyor belt (for solids) or through a microwave-transparent pipe (for liquids and slurries). 


\section{Energy issues in microwave food processing}

\subsection{Review of energy performance studies in microwave food processes}

The enormous magnitude and variety of microwave applications in consumer, commercial and industrial settings creates a strong motivation for improving the energy efficiency of the process. The rapid, selective and volumetric heating capabilities of microwaves are widely acknowledged and often advanced as reasons for their superior energy performance over conventional heaters. Surprisingly, the energy efficiency of microwave-assisted heating processes is still not well-explored (Hoogenboom et al., 2009). Some energy studies on small-scale chemical systems (not more than a few $100 \mathrm{ml}$ or g); e.g. Hoogenboom et al., 2009; Devine and Leadbeater, 2011; Moseley and Kappe, 2011; Morschhäuser et al., 2012; Bermudez et al., 2015 conclude that the energy efficiency of microwave heating systems increase with scale, approaching a saturation value. In the food processing domain, very few energy performance studies on microwave systems are available in the literature. Extensive Scopus and Google Scholar searches featuring the keywords "microwave $x x x$ " and "energy", where xxx refers to various food processes like pasteurisation, sterilisation, cooking, baking, thawing, tempering, frying, etc., hardly reveals any article dedicated to the energy performance of these processes, except perhaps the energy consumption analysis on microwave cooking of rice by Lakshmi et al. (2007). A significant exception to this trend relates to the area of food drying where many relevant studies have been conducted on microwave stand-alone or combination processes. A summary of a representative number of these is presented on Tables 1 and 2. Almost all the studies are concerned with domestic systems of less than 2000W capacity, operating at $2450 \mathrm{MHz}$ or thereabout. A semi-industrial multiple-feed conveyor belt system (Jindarat et al., 2011), with rather large cavity dimensions, consisting of 12 magnetrons operating at $2450 \mathrm{MHz}$, each of which is rated $800 \mathrm{~W}$, with only 6 used in the study, is an exception. The most-significant exception is the $25 \mathrm{~kW}$ capacity, $915 \mathrm{MHz}$ industrial-type system used for one-pass drying of rough rice (Olatunde et al., 2017). Evidently, more research is needed for industrial-scale systems, particularly at 915 (or $896 \mathrm{MHz}$ ), where the penetration depth is about thrice that of the $2450 \mathrm{MHz}$ systems. Aggregating the findings of the various works, it is observed that in general, microwaves, when used alone or in combination with other energy sources, improve overall energy efficiency. Microwave energy performance is maximum at the beginning of drying where the product water loading is maximum, and so, receive maximum power from the microwaves to ensure very fast drying. As drying proceeds, the drop in water loading means more of the incident power will not be absorbed, but reflected, leading to an increase in energy losses.

Most of the studies at variable microwave output power (e.g. Aghilinategh et al., 2015; Alibas, 2007a; Ding et al., 2012; Jafari et al., 2017; Wang et al., 2009; Sharma and Prasad, 2006; Zarein et al., 2015) show that energy performance is improved as microwave power increases, at constant processing mass. An exception is noted in Motevali et al. (2016), where for a microwave power variation from 200W to 900W (in steps of $100 \mathrm{~W}$ ), the energy performance improves up until 400W, after which, it 
declines. Similar trends are seen in Alibas (2007b); and Lule and Koyunku (2017). A possible explanation for these is that for any given drying load, an increase in the microwave power delivery (and hence absorption) leads to an increase in evaporation and heating rates to ensure a more than proportionate output, relative to the increase in power drawn from the system. This must however be balanced by the moisture content of the product. If the moisture level is not high enough, majority of the extra power could be reflected, leading to a drop in system energy performance. Hence, for the same initial moisture level, a maximum power must exist beyond which, reflections will dominate, leading to reduced energy performance. This reasoning is strictly applicable to microwave-only systems. In combination systems, where extra degrees of freedom are added, the situation is no longer as straightforward as extra variables influence system performance. A similar logic can be applied to explain the apparent conflicts in trends observed in systems where for constant microwave power, the load mass is changed. In Gollei et al. (2009); as mass increases, energy performance drops. In Darvishi et al. (2012); energy performance increases with loaded mass until a maximum is attained after which it drops. In Wang et al. (2015a); there is a monotonic increase in energy performance within the mass range of interest (34-200g); and likewise Soysal et al. (2006) in the range 64.3-128.57g. Intermittent power delivery is also shown to improve energy performance in some cases (Yongsawatdigul and Gunasekaran, 1996; Aghilinategh et al., 2015) or worsen it in other cases (e.g. Jindarat et al., 2013). Product properties (e.g. moisture distribution, and form - for instance as bound or free water) would also no doubts have profound effects on energy delivery efficiency. Kurjak et al. (2012) by an imaging technique observed water loss in liquid state, in addition to normal evaporation during the microwave drying of potatoes, apples and onions. This observation, in addition to measured material temperatures, higher than could be derived from the classical heat balance equation, led the researchers to postulate the existence of a "non-thermal" microwave drying effectwhich improves drying rate particularly in the early stages of drying. Overall, mechanistic models are needed to improve understanding of the complex interactions between product properties, processing parameters and efficiency of energy delivery in microwave food drying systems. Also, the general absence of detailed energy studies on the wide variety of microwave food processing applications such as baking, thawing, tempering, pasteurisation, sterilisation, reheating and frying, implies the need for research effort in this area, especially in relation to food quality and safety aspects.

\subsection{Energy performance indices of microwave heating systems}

The energy performance of microwave heating systems is usually quantified either in terms of the specific energy consumption or the energy efficiency. The specific energy consumption is the energy consumption per $\mathrm{kg}$ of processed material in processes other than drying (Lakshmi et al., 2007); or per kg of water evaporated in drying studies (Darvishi et al., 2014). The overall energy efficiency of microwave heating systems is defined (Lakshmi et al., 2007) as the product of the microwave generation efficiency $\eta_{\text {gen }}$ and the microwave absorption efficiency $\eta_{\text {abs, }}$ 
Table 1. Microwave food drying applications in literature: Part 1

\begin{tabular}{|c|c|c|c|c|c|c|c|}
\hline Ref. & Food & $\begin{array}{l}\text { System } \\
\text { type }\end{array}$ & $\begin{array}{l}\text { Cavity } \\
\text { size }\end{array}$ & Frequency & $\begin{array}{l}\text { Power } \\
\text { range }\end{array}$ & $\begin{array}{l}\text { Air \& other } \\
\text { conditions }\end{array}$ & $\begin{array}{l}\text { Specific energy; } \\
\text { Energy efficiency }\end{array}$ \\
\hline $\begin{array}{l}\text { Aghili- } \\
\text { nategh } \\
\text { et al. } \\
(2015)\end{array}$ & Apples & $\begin{array}{l}\text { Microwave- } \\
\text { convective }\end{array}$ & $\begin{array}{l}300 X \\
380 X \\
260 \mathrm{~mm}\end{array}$ & $2465 \mathrm{MHz}$ & $\begin{array}{l}200-600 W \\
\text { duty cycle } \\
16.7-50 \%\end{array}$ & $\begin{array}{l}\text { Air } \\
\text { conditions } \\
1.2 \mathrm{~m} / \mathrm{s} ; \\
40-80 \mathrm{C}\end{array}$ & $\begin{array}{l}\text { Best energy use } \\
@ 600 \mathrm{~W}, \\
22.32 \% \\
\text { duty cycle, 40C, } \\
1.78 \mathrm{~m} / \mathrm{s}\end{array}$ \\
\hline $\begin{array}{l}\text { Alibas } \\
(2007 a)\end{array}$ & $\begin{array}{l}\text { Nettle } \\
\text { leaves }\end{array}$ & $\begin{array}{l}\text { Batch } \\
\text { microwave- } \\
\text { only }\end{array}$ & $\begin{array}{l}327 X \\
370 X \\
207 \mathrm{~mm}\end{array}$ & $2450 \mathrm{MHz}$ & $\begin{array}{l}500,650 \\
750,850 \mathrm{~W}\end{array}$ & & $\begin{array}{l}0.05 \mathrm{kWh} \\
\text { (total, at } 850 \mathrm{~W} \text { ) }\end{array}$ \\
\hline $\begin{array}{l}\text { Alibas } \\
\text { (2007b) }\end{array}$ & $\begin{array}{l}\text { Pumpkin } \\
\text { slices }\end{array}$ & $\begin{array}{l}\text { Microwave- } \\
\text { only \& } \\
\text { convective- } \\
\text { assisted }\end{array}$ & $\begin{array}{l}327 X \\
370 X \\
207 \mathrm{~mm}\end{array}$ & $2450 \mathrm{MHz}$ & $160,350 \mathrm{~W}$ & $\begin{array}{l}1 \mathrm{~m} / \mathrm{s} \\
50,75 \mathrm{C}\end{array}$ & $\begin{array}{l}\text { Stand-alone } \\
\text { most-efficient } \\
(0.23 \mathrm{kWh}) @ \\
160 \mathrm{~W}\end{array}$ \\
\hline $\begin{array}{l}\text { Darvishi } \\
\text { et al. } \\
\text { (2014) }\end{array}$ & $\begin{array}{l}\text { White } \\
\text { mulberry }\end{array}$ & $\begin{array}{l}\text { Batch } \\
\text { microwave- } \\
\text { only }\end{array}$ & $\begin{array}{l}327 X \\
370 X \\
207 \mathrm{~mm}\end{array}$ & $2450 \mathrm{MHz}$ & $100-500 \mathrm{~W}$ & & $\begin{array}{l}3.97-6.73 \\
\mathrm{MJ} / \mathrm{kg}, \\
31.85-55.56 \%\end{array}$ \\
\hline $\begin{array}{l}\text { Darvishi } \\
\text { (2012) }\end{array}$ & $\begin{array}{l}\text { Potato } \\
\text { slices }\end{array}$ & $\begin{array}{l}\text { Batch } \\
\text { microwave- } \\
\text { only }\end{array}$ & $\begin{array}{l}350 X \\
350 x \\
240 \mathrm{~mm}\end{array}$ & $2450 \mathrm{MHz}$ & $\begin{array}{l}\text { Power density } \\
5,10,5,20 \mathrm{~W} / \mathrm{g}\end{array}$ & & $\begin{array}{l}21.38-53.54 \% \text {, } \\
\text { with maximum @ } \\
15 \mathrm{~W} / \mathrm{g}\end{array}$ \\
\hline $\begin{array}{l}\text { Ding } \\
\text { et al. } \\
(2012)\end{array}$ & Daylily & $\begin{array}{l}\text { Microwave- } \\
\text { only }\end{array}$ & $\begin{array}{l}350 X \\
350 X \\
240 \mathrm{~mm}\end{array}$ & $2455 \mathrm{MHz}$ & $\begin{array}{l}\text { 60, 120, } \\
180,240 \mathrm{~W}\end{array}$ & & $\begin{array}{l}\text { High microwave } \\
\text { power, lower } \\
\text { energy use }\end{array}$ \\
\hline $\begin{array}{l}\text { Fang } \\
\text { et al. } \\
\text { (2009) }\end{array}$ & Jujube & $\begin{array}{l}\text { Microwave- } \\
\text { convective (hot } \\
\text { air, then later, } \\
\text { microwave) }\end{array}$ & $\begin{array}{l}350 X \\
350 X \\
240 \mathrm{~mm}\end{array}$ & $2455 \mathrm{MHz}$ & $\begin{array}{l}45,65 \\
90 W\end{array}$ & $\begin{array}{l}\text { Air } 60 \mathrm{C}, \\
0.75 \mathrm{~m} / \mathrm{s} \\
\text { product } \\
\text { moisture } \\
30,40,50 \%\end{array}$ & $\begin{array}{l}\text { Energy savings } \\
55 \% \text { compared to } \\
\text { hot air-only. Put } \\
\text { ON microwave at } \\
\text { higher moistures }\end{array}$ \\
\hline $\begin{array}{l}\text { Gollei } \\
\text { et al. } \\
\text { (2009) }\end{array}$ & Wheat & $\begin{array}{l}\text { Microwave- } \\
\text { only \& } \\
\text { convective- } \\
\text { assisted }\end{array}$ & & $2450 \mathrm{MHz}$ & $800 W$ & $\begin{array}{l}30 \mathrm{~m} 3 / \mathrm{h}, \\
22,45,65 \mathrm{C} \\
2 \mathrm{~kg}, 4 \mathrm{~kg} \\
\text { loading }\end{array}$ & $\begin{array}{l}\text { Best: Hybrid } \\
22.68 \mathrm{MJ} / \mathrm{kg} \\
@ 0.2 \mathrm{~W} / \mathrm{g} \& \\
15.4 \mathrm{MJ} / \mathrm{kg} \\
@ 0.4 \mathrm{~W} / \mathrm{g}\end{array}$ \\
\hline $\begin{array}{l}\text { Hazer- } \\
\text { vazifeh } \\
\text { et al. } \\
(2017) \\
\end{array}$ & $\begin{array}{l}\text { Apple } \\
\text { Slices }\end{array}$ & $\begin{array}{l}\text { Batch } \\
\text { microwave-only }\end{array}$ & $\begin{array}{l}400 X \\
370 X \\
230 \mathrm{~mm}\end{array}$ & $2450 \mathrm{MHz}$ & $\begin{array}{l}500,1000 \\
1500,2000 \mathrm{~W}\end{array}$ & & $\begin{array}{l}\text { Best drying } \\
\text { efficiency } 6.8 \% \\
@ 2000 \mathrm{~W} .>\text { that } \\
\text { of hot air drying }\end{array}$ \\
\hline $\begin{array}{l}\text { Holtz } \\
\text { et al. } \\
\text { (2010) }\end{array}$ & $\begin{array}{l}\text { Swede, } \\
\text { Potato, } \\
\text { bread }\end{array}$ & $\begin{array}{l}\text { Batch } \\
\text { microwave- } \\
\text { convective }\end{array}$ & & $2450 \mathrm{MHz}$ & $300 \mathrm{~W}$ & $50 \mathrm{C} ; 4.5 \mathrm{~m} / \mathrm{s}$ & $\begin{array}{l}\text { SEC 15-18 } \\
\text { MJ/kg-electrical } \\
\text { (for all products) }\end{array}$ \\
\hline $\begin{array}{l}\text { Wang } \\
\text { et al. } \\
\text { (2015a) }\end{array}$ & $\begin{array}{l}\text { Cotton } \\
\text { stalks }\end{array}$ & $\begin{array}{l}\text { Batch } \\
\text { microwave- } \\
\text { only }\end{array}$ & & $2450 \mathrm{MHz}$ & $1000 \mathrm{~W}$ & $\begin{array}{l}\text { Different } \\
\text { loading: } \\
\text { 34-200g }\end{array}$ & $\begin{array}{l}\text { Drying efficiency } \\
7.52-19.78 \% \\
\text { Specific energy } \\
12.49-35.9 \mathrm{MJ} / \mathrm{kg} \\
\text { (best @ 200g) }\end{array}$ \\
\hline $\begin{array}{l}\text { Jafari } \\
\text { et al. } \\
\text { (2017) }\end{array}$ & Paddy & $\begin{array}{l}\text { Semi- } \\
\text { continuous } \\
\text { microwave- } \\
\text { only }\end{array}$ & & $2450 \mathrm{MHz}$ & $\begin{array}{l}90,270 \\
450 W\end{array}$ & $\begin{array}{l}6,9,18 \mathrm{~mm} \\
\text { bed } \\
\text { thicknesses }\end{array}$ & $\begin{array}{l}\text { Higher } \\
\text { microwave } \\
\text { power, lower } \\
\text { energy use }\end{array}$ \\
\hline $\begin{array}{l}\text { Jiang } \\
\text { et al. } \\
\text { (2017) }\end{array}$ & $\begin{array}{l}\text { Okra } \\
\text { snack }\end{array}$ & $\begin{array}{l}\text { Freeze } \\
\text { drying+ } \\
\text { microwave- } \\
\text { vacuum }\end{array}$ & & $2450 \mathrm{MHz}$ & $\begin{array}{l}1536 \mathrm{~W} \\
\text { pulsed } \\
66.7 \% \text { duty } \\
\text { cycle }\end{array}$ & $\begin{array}{l}1^{\text {st }} \text { freeze } \\
\text { dry, } 10 \mathrm{hrs} \\
@ 70 \mathrm{kPa} \text { to } \\
80 \% \text { water }\end{array}$ & $\begin{array}{l}71.92 \% \text { energy } \\
\text { savings relative } \\
\text { to normal freeze } \\
\text { drying }\end{array}$ \\
\hline $\begin{array}{l}\text { Jindarat } \\
\text { et al. } \\
\text { (2011) }\end{array}$ & - & $\begin{array}{l}\text { Continuous belt } \\
\text { microwave- } \\
\text { convective }\end{array}$ & $\begin{array}{l}90 X \\
45 X \\
270 \mathrm{~cm}\end{array}$ & $2450 \mathrm{MHz}$ & $\begin{array}{l}8000 \mathrm{~W} \times 6 \\
=4800 \mathrm{~W}\end{array}$ & $\begin{array}{l}0.5 \mathrm{~m} / \mathrm{s} \\
30,50,70 \mathrm{C}\end{array}$ & $\begin{array}{l}\text { Energy } \\
\text { Efficiency }=40 \%\end{array}$ \\
\hline
\end{tabular}


Table 2. Microwave food drying applications in literature: Part 2

\begin{tabular}{|c|c|c|c|c|c|c|c|}
\hline Ref. & Food & $\begin{array}{l}\text { System } \\
\text { type }\end{array}$ & $\begin{array}{l}\text { Cavity } \\
\text { size }\end{array}$ & Frequency & $\begin{array}{l}\text { Power } \\
\text { range }\end{array}$ & $\begin{array}{l}\text { Air \& other } \\
\text { conditions }\end{array}$ & $\begin{array}{l}\text { Specific energy; } \\
\text { Energy efficiency }\end{array}$ \\
\hline $\begin{array}{l}\text { Jindarat } \\
\text { et al. } \\
(2013)\end{array}$ & $\begin{array}{l}\text { Tea } \\
\text { Leaves }\end{array}$ & $\begin{array}{l}\text { Double-feed } \\
\text { microwave+vacuum }\end{array}$ & & $2450 \mathrm{MHz}$ & $\begin{array}{l}800, \\
1600 w \\
\text { continuous } \\
\& \text { pulsed } \\
\text { (50\% duty } \\
\text { cycle) } \\
\end{array}$ & $\begin{array}{l}\text { Vacuum: } \\
71.3 \& \\
51.3 \mathrm{kPa}\end{array}$ & $\begin{array}{l}\text { Optimal @ 800W } \\
\text { continuous } \\
51.3 \mathrm{kPa}\end{array}$ \\
\hline $\begin{array}{l}\text { Khoshta- } \\
\text { ghaza } \\
\text { et al. } \\
(2015) \\
\end{array}$ & $\begin{array}{l}\text { Soya- } \\
\text { bean } \\
\text { kernel }\end{array}$ & $\begin{array}{l}\text { Microwave } \\
+ \text { fluidized } \\
\text { bed hot air }\end{array}$ & & $2450 \mathrm{MHz}$ & $200-500 W$ & $\begin{array}{l}80-140 \mathrm{C} \\
1.8-4.5 \mathrm{~m} / \mathrm{s}\end{array}$ & $\begin{array}{l}\text { Optimal @ 500W } \\
80 \mathrm{C}, 4.5 \mathrm{~m} / \mathrm{s}\end{array}$ \\
\hline $\begin{array}{l}\text { Kurjak } \\
\text { et al. } \\
(2012)\end{array}$ & $\begin{array}{l}\text { Potato, } \\
\text { apple, } \\
\text { onion }\end{array}$ & $\begin{array}{l}\text { Batch } \\
\text { microwave- } \\
\text { only }\end{array}$ & & $2450 \mathrm{MHz}$ & $456,523 \mathrm{~W}$ & & $\begin{array}{l}\text { Microwave energy } \\
\text { for Onion>Apple> } \\
\text { Potato }\end{array}$ \\
\hline $\begin{array}{l}\text { Lule } \\
\text { and } \\
\text { Koyuncu } \\
\text { (2017) }\end{array}$ & $\begin{array}{l}\text { Nettle } \\
\text { leaves }\end{array}$ & $\begin{array}{l}\text { Batch } \\
\text { microwave- } \\
\text { only }\end{array}$ & & $2450 \mathrm{MHz}$ & $\begin{array}{l}90,160, \\
350 \mathrm{~W}\end{array}$ & & $\begin{array}{l}\text { Best @ 160W } \\
23.63 \text { kWh/kg }\end{array}$ \\
\hline $\begin{array}{l}\text { Motevali } \\
\text { et al. } \\
(2011)\end{array}$ & $\begin{array}{l}\text { Mush- } \\
\text { room } \\
\text { slices }\end{array}$ & $\begin{array}{l}\text { Batch microwave- } \\
\text { only \& } \\
\text { microwave-vacuum }\end{array}$ & $\begin{array}{l}230 X \\
320 X \\
360 \mathrm{~mm}\end{array}$ & $2450 \mathrm{MHz}$ & $\begin{array}{l}130,260, \\
380, \\
450 W\end{array}$ & $\begin{array}{l}20,40,60 \\
80 \mathrm{kPa}\end{array}$ & $\begin{array}{l}\text { Best: } 0.28 \\
\text { kWh @ 450w \& } \\
0.14 \mathrm{kWh} @ \\
450 \mathrm{~W}, 20 \mathrm{kPa}\end{array}$ \\
\hline $\begin{array}{l}\text { Motevali } \\
\text { et al. } \\
(2016)\end{array}$ & $\begin{array}{l}\text { Chamo- } \\
\text { mile }\end{array}$ & $\begin{array}{l}\text { Batch } \\
\text { microwave- } \\
\text { convective }\end{array}$ & $\begin{array}{l}\text { Cylinder } \\
90 \times \\
380 \mathrm{~mm}\end{array}$ & $2450 \mathrm{MHz}$ & $\begin{array}{l}\text { 200-900W } \\
\text { (steps of } \\
100 W \text { ) }\end{array}$ & $0.5 \mathrm{~m} / \mathrm{s}, 50 \mathrm{C}$ & $\begin{array}{l}\text { 18.93-28.15 } \\
\mathrm{MJ} / \mathrm{kg} @ 400 \mathrm{~W} \\
8.25-13.07 \%\end{array}$ \\
\hline $\begin{array}{l}\text { Mousa } \\
\text { and } \\
\text { Farid } \\
(2007)\end{array}$ & $\begin{array}{l}\text { Banana } \\
\text { slices }\end{array}$ & $\begin{array}{l}\text { Batch } \\
\text { microwave } \\
\text { vacuum }\end{array}$ & $\begin{array}{l}330 X \\
350 X \\
240 \mathrm{~mm}\end{array}$ & $2450 \mathrm{MHz}$ & $\begin{array}{l}600 W \\
\text { pulsed } \\
28 \% \\
\text { duty cycle } \\
\end{array}$ & $\begin{array}{l}\text { Vacuum: } \\
\text { 101, } \\
50 \& 30 \mathrm{kPa}\end{array}$ & $\begin{array}{l}\text { 100-40\% } \\
\text { with vacuum; } \\
100-20 \% \\
\text { without }\end{array}$ \\
\hline $\begin{array}{l}\text { Olatunde } \\
\text { et al. } \\
(2017)\end{array}$ & $\begin{array}{l}\text { Rough } \\
\text { rice }\end{array}$ & $\begin{array}{l}\text { Continuous } \\
\text { belt } \\
\text { microwave- } \\
\text { only }\end{array}$ & & $915 \mathrm{MHz}$ & $\begin{array}{l}3000- \\
25000 W\end{array}$ & $\begin{array}{l}96-480 \mathrm{~kg} ; \\
100-500 \mathrm{~cm} \\
\text { bed } \\
\text { thicknesses }\end{array}$ & $\begin{array}{l}4810 \mathrm{~kJ} / \mathrm{kg} @ \\
500 \mathrm{~cm}, 15 \mathrm{~kW} \\
5985 \mathrm{~kJ} / \mathrm{kg} @ \\
100 \mathrm{~cm}, 5 \mathrm{~kW}\end{array}$ \\
\hline $\begin{array}{l}\text { Sharma } \\
\text { and } \\
\text { Prasad } \\
(2006)\end{array}$ & $\begin{array}{l}\text { Garlic } \\
\text { cloves }\end{array}$ & $\begin{array}{l}\text { Batch } \\
\text { microwave- } \\
\text { convective }\end{array}$ & & $2450 \mathrm{MHz}$ & $\begin{array}{l}10,20 \\
30,40 \mathrm{~W}\end{array}$ & & $\begin{array}{l}\text { 70\% energy } \\
\text { savings @ 40w }\end{array}$ \\
\hline $\begin{array}{l}\text { Soysal } \\
\text { et al. } \\
(2006)\end{array}$ & Parsley & $\begin{array}{l}\text { Batch } \\
\text { microwave- } \\
\text { only }\end{array}$ & $\begin{array}{l}215 X \\
350 X \\
330 \mathrm{~mm}\end{array}$ & $2450 \mathrm{MHz}$ & $\begin{array}{l}900 W \\
\text { pulsed } \\
50 \% \\
\text { duty cycle }\end{array}$ & $\begin{array}{l}\text { Loading: } \\
64.3- \\
128.57 \mathrm{~g}\end{array}$ & $\begin{array}{l}9.5 \% \text { rise in drying } \\
\text { efficiency } \\
\text { with mass }\end{array}$ \\
\hline $\begin{array}{l}\text { Varith } \\
\text { et al. } \\
\text { (2007) }\end{array}$ & Longan & $\begin{array}{l}\text { Microwave- } \\
\text { convective }\end{array}$ & & $2450 \mathrm{MHz}$ & $\begin{array}{l}100,180 \\
300,450 W\end{array}$ & $\begin{array}{l}40,50,60 \mathrm{C} \\
0.7 \mathrm{mls}\end{array}$ & $\begin{array}{l}\text { 48.2\% energy } \\
\text { savings @ } \\
\text { Optimal: } 40 \mathrm{C} \text {, } \\
450 \mathrm{~W} \text { for } 1.7 \mathrm{~h} \\
\text { then } 60 \mathrm{C} \& \\
300 \mathrm{~W} \text { for } 3.3 \mathrm{~h}\end{array}$ \\
\hline $\begin{array}{l}\text { Wang } \\
\text { et al. } \\
\text { (2009) }\end{array}$ & Jujube & $\begin{array}{l}\text { Microwave- } \\
\text { only }\end{array}$ & $\begin{array}{l}350 X \\
350 X \\
240 \mathrm{~mm}\end{array}$ & $2455 \mathrm{MHz}$ & $\begin{array}{l}45,90 \\
135 \mathrm{~W}\end{array}$ & & $\begin{array}{l}\text { Energy optimal } \\
\text { @ } 135 \mathrm{~W}\end{array}$ \\
\hline $\begin{array}{l}\text { Yongsa- } \\
\text { watdigul } \\
\text { and } \\
\text { Gunase- } \\
\text { karan } \\
(1996)\end{array}$ & $\begin{array}{l}\text { Cran- } \\
\text { berries }\end{array}$ & $\begin{array}{l}\text { Continuous } \\
\text { microwave- } \\
\text { vacuum }\end{array}$ & & $2450 \mathrm{MHz}$ & $\begin{array}{l}\text { Continuous } \\
(250, \\
500 W) \\
\text { Pulsed } \\
250 w \text { @ } \\
\text { varied duty } \\
\text { cycles }\end{array}$ & $\begin{array}{l}\text { Vacuum } \\
10.67,5.33 \\
\mathrm{kPa}\end{array}$ & $\begin{array}{l}\text { Optimal } 2.66 \\
\mathrm{MJ} / \mathrm{kg} \text { pulsed } \\
(250 \mathrm{~W}) \text { at } \\
16.7 \% \text { duty cycle } \\
\& 5.33 \mathrm{kPa}\end{array}$ \\
\hline $\begin{array}{l}\text { Zarein } \\
\text { et al. } \\
\text { (2015) }\end{array}$ & $\begin{array}{l}\text { Apple } \\
\text { slices }\end{array}$ & $\begin{array}{l}\text { Batch } \\
\text { microwave- } \\
\text { only }\end{array}$ & & $2450 \mathrm{MHz}$ & $\begin{array}{l}200,400 \\
600 W\end{array}$ & & $\begin{array}{l}\text { Max: } 54.34 \% \\
@ 600 W ; \\
\text { Min 17.42\% } \\
@ \text { 200W }\end{array}$ \\
\hline
\end{tabular}


where (for $P_{o u t, m i c r}=$ microwave output power; $P_{i n, e l e c t}=$ input electrical power; $P_{\text {heat }}=$ thermal power delivered),

$\eta_{\text {gen }}=\frac{P_{\text {out } \text { micr }}}{P_{\text {in, elect }}}$

$\eta_{\text {abs }}=\frac{P_{\text {heat }}}{P_{\text {out, micr }}}$

Generation efficiency largely depends on the quality of the microwave power source (i.e. magnetron), while absorption efficiency depends on the degree of impedance matching between the source and the processed food (load) - which also depends on the load's dielectric properties. When computing efficiencies (2) and (3), it is important that the microwave output power is based on real-time measurements rather than prior manufacturers' ratings and settings. This is because the magnetron output power delivery declines with age and related factors - which will lead to overestimation of generation efficiencies and underestimation of absorption efficiencies. Output drops by as much as 33\% have been reported after 5-10 years of operation (Anantheswaran, 1999). Even within 30 minutes of continuous operation, output drops in the order of $15 \%$ are common in domestic microwave ovens (Swain et al., 2006). Little wonder, electrical-to-microwave conversion efficiencies as low as $50-60 \%$ are common (Lakshmi et al., 2007; Wang et al., 2015b), even though quoted magnetron efficiencies are often higher (over $70 \%$ for $2450 \mathrm{MHz}$, and $88 \%$ for $915 \mathrm{MHz}$ systems). The effects of any error in microwave power estimation are cancelled out in the overall efficiency computation (1). However, care must be taken to subtract standby power consumption obtained by performing no-load tests (Gollei et al., 2009; Wang et al., 2015b) from the total power drawn from the mains, when the entire system is on. The standby power is the power consumed when the system and all auxiliary components like coolers, fans, etc. are switched on, but the magnetron is off.

\subsection{Energy performance indices of microwave drying systems: the need for unification and standardisation}

Microwave drying processes are different from other microwave heating processes in that the incident power is deliberately converted not only to sensible heat, but latent heat of evaporation. Energy performance is maximum at the beginning of drying, declining as the water load diminishes; and hence, are normally expressed as timeaveraged values. Most of the studied works on energy performance of microwavebased drying systems have expressed specific energy consumption as total electrical energy consumed (including those of auxiliaries like fans, belt drives and pumps) per $\mathrm{kg}$ of water evaporated. As auxiliary systems vary remarkably across installations, it is very difficult to compare results from different systems. Some studies (e.g. Yongsawatdigul \& Gunasekaran, 1996; Aghilinategh et al., 2015), have however 
expressed it in terms of thermal energy only, neglecting electrical aspects. The energy (thermal) efficiency of microwave drying systems (from the studies detailed in Tables 1 \& 2), have in general, been expressed as

$$
\eta_{\text {micrD , thermal }}=\frac{Q_{\text {sens }}+Q_{\text {lat }}}{Q_{\text {micr,out }}+Q_{\text {comb }}}
$$

where Qsens is the sensible heat used in heating up the product (and the water therein) from the feed temperature to the evaporating temperature and $Q_{\text {lat, }}$ the latent heat of the evaporated moisture, at the evaporating temperature. Qmicr,out is the output microwave power integrated over operating time; while $\mathrm{Q}_{\text {comb }}$ is the energy spent in the assisting or combination process(es), where they exist. A related term "drying efficiency" (Soysal et al., 2006; Wang et al., 2015a), sometimes used interchangeably with "thermal or energy efficiency" discounts the sensible heat term from (4) that is:

$$
\eta_{\text {micrD , drying }}=\frac{Q_{\text {lat }}}{Q_{\text {micr, out }}+Q_{\text {comb }}}
$$

The conventional drying literature however has slightly different meanings for the terms "energy or thermal efficiency" as well as "drying efficiency". Thermal efficiency is defined in the traditional drying literature (Kudra, 2004; Menshutina et al., 2004; Atuonwu et al., 2012) as the ratio of latent heat delivered (evaluated at feed temperature) to the total thermal energy input. Comparing this with the equation for the same term, in microwave drying literature (equation 4), it is observed that the sensible heat term $\mathrm{Q}_{\text {sens }}$ is missing, from the conventional drying expression. This is a major discrepancy. However, since the evaporating temperature is higher than the feed temperature (which necessitates the supply of $\mathrm{Q}_{\text {sens }}$ in the first place in equation (4)), the latent heat at the evaporating temperature (as used in (4)) is lower than at the feed temperature (as used in the conventional drying literature). Hence, the omission of Qsens in conventional drying would mean that the results obtained from the two calculations will be close to each other, but not exactly the same.

The thermal efficiency in the conventional drying literature is about the same as the "drying efficiency" (equation (5)) in the microwave drying literature-but with the latent heat evaluated at the evaporating temperature in the latter case. Drying efficiency in the traditional drying literature (Kudra, 2004; Menshutina et al., 2004) however discounts the energy of the dryer exhaust air from the total thermal energy input (the denominator), and so, is considerably different from "drying efficiency" as defined in microwave drying systems. A unification of energy performance indices is thus recommended across board, to facilitate comparison.

\subsection{Energy standards and regulations}

The microwave power output of domestic $2450 \mathrm{MHz}$ microwave ovens (shown in labels), have traditionally been calibrated based on the IEC 60705 standards (equivalent to the EN 60705). The calorimetry-based calibration method (IEC 60705, 
1999) involves heating $1000 \mathrm{~g} \pm 5 \mathrm{~g}$ of water, inside a cylindrical borosilicate glass container (external diameter $190 \mathrm{~mm}$, height $90 \mathrm{~mm}$ ), from $10^{\circ} \mathrm{C} \pm 1^{\circ} \mathrm{C}$ to $20^{\circ} \mathrm{C} \pm 2^{\circ} \mathrm{C}$. The microwave power output is then calculated as

$$
P_{\text {out }, \text { micr }}=\frac{4.187 m_{W}\left(T_{2}-T_{1}\right)+0.55 m_{C}\left(T_{2}-T_{0}\right)}{t}
$$

where $m_{W}$ is the mass of the water; $m_{C}$, the mass of the container; $T_{0}$, the ambient temperature; $T_{1}$ and $T_{2}$, the initial and final temperatures of the water, respectively. The corresponding energy consumption $W_{\text {in }}$ (electrical input power aggregated over time $t$ ), is measured, from which the microwave generation efficiency is determined as $\mathrm{P}_{\text {out,mirr }} / \mathrm{W}$ in. The choice of the operating conditions in the calorimetric experiments, is based on minimising heat loss and container heat capacity effects (IEC 60705, 1999), as well as obtaining a matched load condition for maximum microwave power transfer to heat (without reflections). Poutmicr is thus good as a standard against which thermal energy delivery to any load, can be compared, to determine absorption efficiency (equation 3). The corresponding mains power $W_{i n}$, is however not indicative of system energy consumption under actual normal usage conditions - variable loads (shape \& mass) and much higher temperature differentials. FSP (2007), for instance, classified all kinds of heating done in microwave ovens into 6 categories (from simple reheating of light ready meals, to the cooking of heavy meals from raw state)-See Table 3. In all these, the required temperature rise, ranged from a minimum of $30^{\circ} \mathrm{C}$ to a maximum of over $125^{\circ} \mathrm{C}$, as against the $10^{\circ} \mathrm{C}$ used in the IEC standards. A more usage-reflective standard energy consumption indicator was thus required. To tackle this challenge, it was advocated in HEECS (2011) to

i. Create a method that is representative of a real use of the microwave function in microwave ovens that is, reheating readymade food portions, beverages/liquids defrosting food and preparing meals from raw states.

ii. Ensure the use of an easy-to-find test load whose properties are identical and independent of geographical location and season of the year. The weight and shape of this test load shall have a good correlation to real use.

iii. The method must fulfil requirements of repeatability and reproducibility (crucial for energy measuring purposes), and allow for differentiation in terms of energy consumptions among products.

It was suggested to use water test loads as before, but in three different masses, in different-shaped containers, and two temperature rises (both in the order of $50^{\circ} \mathrm{C}$ ). The measured energy usage in these different cases will be weighted and averaged, to approximate true usage conditions. This was implemented in the latest Standards IEC 60705, 2010 (same as BS EN 60705, 2015). Three masses of water $(1000 \mathrm{~g} \pm 1 \mathrm{~g}$, $350 \mathrm{~g} \pm 1 \mathrm{~g}$, and $275 \mathrm{~g} \pm 1 \mathrm{~g}$ ), were used in cylindrical containers of capacities $2000 \mathrm{ml}$ (diameter $190 \mathrm{~mm} \pm 1 \mathrm{~mm}$, height $90 \mathrm{~mm} \pm 1 \mathrm{~mm}$ ), $900 \mathrm{ml}$ (diameter $140 \mathrm{~mm} \pm 1 \mathrm{~mm}$, height $76 \mathrm{~mm} \pm 1 \mathrm{~mm}$ ) and $600 \mathrm{ml}$ (diameter $125 \mathrm{~mm} \pm 1 \mathrm{~mm}$, height $90 \mathrm{~mm} \pm 1 \mathrm{~mm}$ ), respectively. Each was heated from $10^{\circ} \mathrm{C} \pm 0.5^{\circ} \mathrm{C}$ to between 60 and $65^{\circ} \mathrm{C}$ in the first case, and then 
to between 55 and $60^{\circ} \mathrm{C}$ in the second. The overall measured energies for the $1000 \mathrm{~g}$, $350 \mathrm{~g}$ and $275 \mathrm{~g}$ cases were then weighted in the ratio $3: 6: 2$, based on research findings on domestic loads, which show a statistical bias towards the $350 \mathrm{~g}$ range. Detailed descriptions of the method and the formulae used is presented in BS EN 60705 (2015). The final result is designated as $W_{50}$, the "energy consumption of a cooking cycle to reach a temperature increase of $50 \mathrm{~K}$ ". Such standards, specific to industrial microwave heaters (896 or $915 \mathrm{MHz}$ ) systems are not available in the literature.

To encourage energy-efficiency, energy labelling schemes have widely been employed for a wide variety of energy-related products or appliances. While this is true for microwave ovens in some countries, like Brazil, China and Japan (see Tables 4, 5 and 6), the same cannot be said for the European Union (EC, 2017). It is however expected that the improved microwave oven energy consumption and efficiency test methods (as detailed in the foregoing), would enable future enable energy labelling for microwave ovens in the European Union, being more representative of actual usage conditions than the standard IEC power calibration tests.

Table 3: Categories of microwave heating of various foods at typical final temperatures FSP (2007).

\begin{tabular}{|c|c|c|c|c|}
\hline Category & Food item & Type & $\begin{array}{c}\text { Starting } \\
\text { temperature }\left({ }^{\circ} \mathrm{C}\right)\end{array}$ & $\begin{array}{c}\text { Final } \\
\text { temperature } \\
\text { range }\left({ }^{\circ} \mathrm{C}\right)\end{array}$ \\
\hline \multirow[t]{4}{*}{1} & Crackers & \multirow[b]{2}{*}{ Light product } & Room temp. & \multirow[b]{4}{*}{$49-66$} \\
\hline & Bread & & Room temp. & \\
\hline & Sandwich & \multirow[b]{2}{*}{ Heavy product } & Room temp. & \\
\hline & Muffins or cake & & Room temp. & \\
\hline \multirow[t]{4}{*}{2} & Waffles & \multirow[b]{2}{*}{ Light product } & Frozen $\leq-18$ & \multirow[b]{4}{*}{$54-82$} \\
\hline & Pancakes & & Frozen $\leq-18$ & \\
\hline & Broccoli & \multirow[b]{2}{*}{ Heavy product } & Frozen $\leq-18$ & \\
\hline & Cauliflower & & Frozen $\leq-18$ & \\
\hline \multirow[t]{4}{*}{3} & Cinnamon rolls & \multirow[b]{2}{*}{ Light product } & Frozen $\leq-18$ & \multirow[b]{4}{*}{$71-96$} \\
\hline & Melting cheese & & Refrigerated $\leq-4$ & \\
\hline & Pizza & \multirow{2}{*}{ Heavy product } & Frozen $\leq-18$ & \\
\hline & Pre-cooked hamburger patties & & Frozen $\leq-18$ & \\
\hline \multirow[t]{4}{*}{4} & Pre-cooked pork sausage & \multirow[b]{2}{*}{ Light product } & Frozen $\leq-18$ & \multirow[b]{4}{*}{$77-104$} \\
\hline & Melting butter or margarine & & Refrigerated $\leq-4$ & \\
\hline & Lasagne & \multirow{2}{*}{ Heavy product } & Frozen $\leq-18$ & \\
\hline & Macaroni and cheese & & Frozen $\leq-18$ & \\
\hline \multirow[t]{2}{*}{5} & Melting chocolate squares & Light product & Room temp. & \multirow[b]{2}{*}{$77-121$} \\
\hline & Vegetables with cheese sauce & Heavy product & Frozen $\leq-18$ & \\
\hline \multirow[t]{2}{*}{6} & Melting caramels & Light product & Room temp. & \multirow[b]{2}{*}{$77->121$} \\
\hline & Sliced bacon & Heavy product & Refrigerated $\leq-4$ & \\
\hline
\end{tabular}

Table 4. Energy labelling of microwave ovens, Brazil, based on IEC 60705 (1999) -HEECS (2011)

\begin{tabular}{|c|c|}
\hline Class & Energy efficiency indices \\
\hline A & Efficiency $\geq 54 \%$ \\
\hline B & $49 \% \leq$ Efficiency $<54 \%$ \\
\hline C & $45 \%<$ Efficiency $<49 \%$ \\
\hline
\end{tabular}


Table 5. Energy labelling of microwave ovens, China, based on IEC 60705 (1999) -HEECS (2011)

\begin{tabular}{|c|c|}
\hline Energy-efficiency grades & Efficiency values (\%) \\
\hline 1 & 62 \\
\hline 2 & 60 \\
\hline 3 & 58 \\
\hline 4 & 56 \\
\hline 6 & 54 \\
\hline
\end{tabular}

Table 6. Energy labelling of microwave ovens, Japan, based on the "Top Runner" program -MOE (2015)

\begin{tabular}{|c|c|c|c|c|}
\hline \multicolumn{4}{|c|}{ Category } & \multirow{2}{*}{$\begin{array}{c}\text { Standard energy } \\
\text { consumption } \\
\text { efficiency (\%) }\end{array}$} \\
\hline Function & Heating method & $\begin{array}{l}\text { Internal } \\
\text { volume }\end{array}$ & $\begin{array}{l}\text { Category } \\
\text { name }\end{array}$ & \\
\hline $\begin{array}{l}\text { Single-function microwave } \\
\text { (with no oven function) }\end{array}$ & - & - & A & 60.1 \\
\hline \multirow{5}{*}{$\begin{array}{l}\text { Microwave with oven } \\
\text { function (oven ranges) }\end{array}$} & \multirow{2}{*}{$\begin{array}{l}\text { With an exposed } \\
\text { heater (excluding } \\
\text { those with hot air } \\
\text { circulation heating) }\end{array}$} & $<30$ litres & B & 73.4 \\
\hline & & $\geq 30$ litres & C & 78.2 \\
\hline & \multirow{2}{*}{$\begin{array}{l}\text { Without an exposed } \\
\text { heater (excluding } \\
\text { those with hot air } \\
\text { circulation heating) }\end{array}$} & $<30$ litres & D & 70.4 \\
\hline & & $<30$ litres & $E$ & 79.6 \\
\hline & $\begin{array}{c}\text { With hot air } \\
\text { circulation heating }\end{array}$ & - & $\mathrm{F}$ & 73.5 \\
\hline
\end{tabular}

\subsection{Factors affecting energy-efficiency}

From equations (1) - (3), the overall energy efficiency (1) is affected by

i. The source side (microwave generator to the transmitting waveguide), which determines the generation efficiency (equation 2)

ii. The load side (the processed food and the cavity housing it), which determines the absorption efficiency (equation 3)

iii. The matching between the source and load sides (affecting equation (3)).

Since the inception of the use of microwaves in heating applications some seven decades ago, they have always been powered by vacuum electronic devices such as magnetrons, klystrons and gyrotrons. Of these, magnetrons constitute the vast majority being the cheapest and most-efficient (Edgar and Osepchuk, 2001). They have a maximum generation efficiency (equation 2) of about $70 \%$ (at $2450 \mathrm{MHz}$ ), and $88 \%$ (at $915 \mathrm{MHz}$ ). The absorption efficiency is however determined by the degree of impedance matching between the source and load. The load impedance varies with time, as the food temperature changes. It also varies with space due to the inhomogeneity in thermal \& dielectric properties as well as the non-uniform spatial temperatures. As a result of these, mismatch is bound to occur, leading to reflections and reduced absorption efficiency. Overall efficiencies (equation 1) are therefore usually in the order of $25-55 \%$, depending on load size (Wang et al., 2015b). Nonuniform heating implies that for the coldest spots to reach the optimal temperature 
necessary for food safety, higher than optimal temperatures must be delivered to the hottest spots, incurring both quality and energy penalties. Atuonwu and Tassou (2017) analysed the energy implications of non-uniform heating, deriving equations connecting the maximum percentage energy savings associated with improving the heating uniformity of microwave processes. Depending on specific processing conditions, energy savings in the order of $20 \%$ were shown to be possible for pasteurisation and sterilisation processes, while about $85 \%$ savings is achievable for thawing processes where the non-uniformity is very severe. From the foregoing it becomes necessary to have a means of controlling the source and load variables to minimise reflections (or maximise absorption efficiency). Magnetrons fail severely in this, due to their poor flexibility and feedback controllability. Solid-state microwave power generators have however proved capable of receiving feedback on the dynamic states of the load and controlling the source power delivery to match with the needs of the load, using control inputs like phase, frequency and power amplitude. These capabilities are further enhanced in systems with multiple generators. Therefore, the next sections of this article will focus on the possibilities and challenges (from energy and cost perspectives), of deploying solid-state sources as alternative to magnetrons.

\section{Solid-state power delivery for process energy-efficiency}

\subsection{Principle of operation}

A solid-state power microwave heating system as shown in Fig. 4 consists of a microwave small-signal generator, a high power amplifier (which replaces the magnetron), with its heat sink, and a power supply system. The small-signal generator is essentially an oscillator that generates a low-amplitude microwave signal, which is sent to the input of the power amplifier, to which a DC power supply has been applied. The DC power supply consists of a step-down transformer which reduces the mains $A C$ voltage to the required value, a rectifier circuit which converts the $A C$ to a pulse of single polarity, and a filter circuit which converts the pulsed signal to pure DC (in the order of 50V). The power amplifier, in receiving the DC power, converts the input lowamplitude microwave signal to a higher amplitude microwave signal at the same frequency $\left(R F_{\text {out }}\right.$ ), which is then channelled to the heating cavity. This power amplifier is essentially a power transistor (semi-conductor device consisting of three semiconductor materials, joined together in two junctions). From these three semiconductor materials, three terminals are drawn out for connection to an external circuit. The three semiconductor materials are P-type and N-type materials, hence, based on material composition, there are PNP and NPN transistors. P-type materials are formed by adding impurities (called dopants) to pure semiconductor materials like silicon or germanium, to create electron deficits; while N-type materials are doped to have electron excess. There are different types of transistor constructions, the most popular being the bipolar junction and field effect transistors. An in-depth discussion on transistors is available in Bahl (2009). Fig. 5 shows a block diagram and circuit configuration of the simplest commonly-used transistor circuit form: an NPN transistor in common-emitter mode. As long as a small signal voltage (RF input, with any necessary biasing), applied at the base of the transistor is able to drive it into the so- 
called active region of its characteristics, the resultant small current flowing through the base is amplified by an amplification factor $\beta$ (sometimes as high as 200). Also, the voltage is amplified approximately by $\beta R_{c} / R_{b}$ where $R_{c}=$ collector circuit output resistance and $R_{b}=$ base circuit input resistance. The power gain $\beta^{2} R_{d} / R_{b}$ from $R F$ input to RF output is therefore very high. The output voltage is limited by the power supply voltage $V_{c c}$, while the output current is a measure of the $A C$ current drawn from the mains. Hence, while the microwave power output is much larger than the microwave power input, it does not exceed the DC power applied (and by extension, the AC power drawn from the mains). The power conversion is commonly quantified by the drain and power-added efficiencies, given respectively by

$$
\eta_{D}=\frac{R F_{\text {out }}}{P_{\mathrm{dc}, \text { in }}}
$$

$\eta_{P A}=\frac{R F_{\text {out }}-R F_{\text {in }}}{P_{\mathrm{dc}, \text { in }}}=\frac{G}{G-1} \eta_{D}$

where the decibel power gain $G d B$ is

$$
G d B=10 \log (G)=10 \log \left(\frac{R F_{\text {out }}}{R F_{\text {in }}}\right) \approx 10 \log \left(\beta^{2} R_{c} / R_{b}\right)
$$

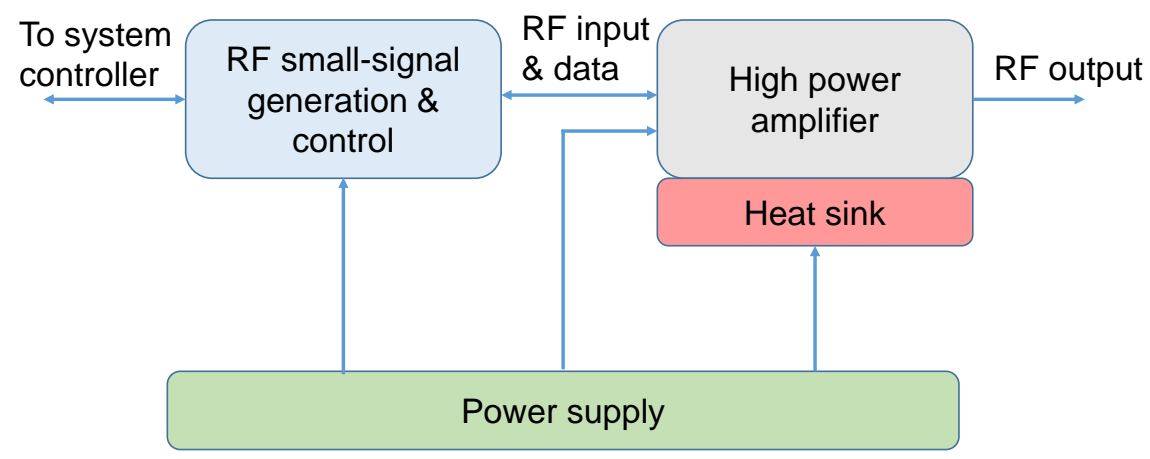

Fig. 4. Block diagram of solid-state microwave generator system (Werner, 2015) 


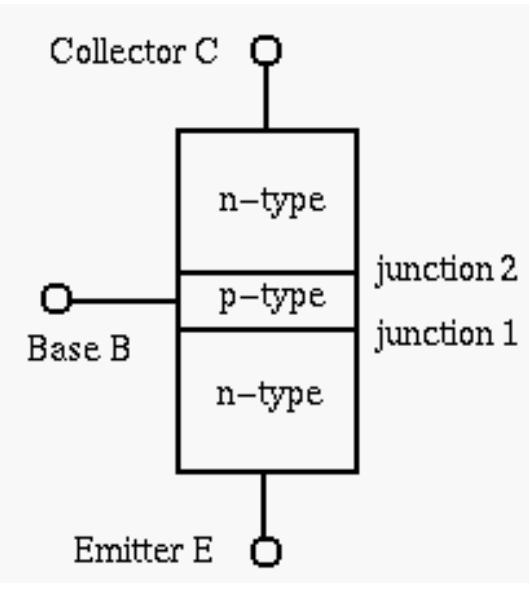

(a)

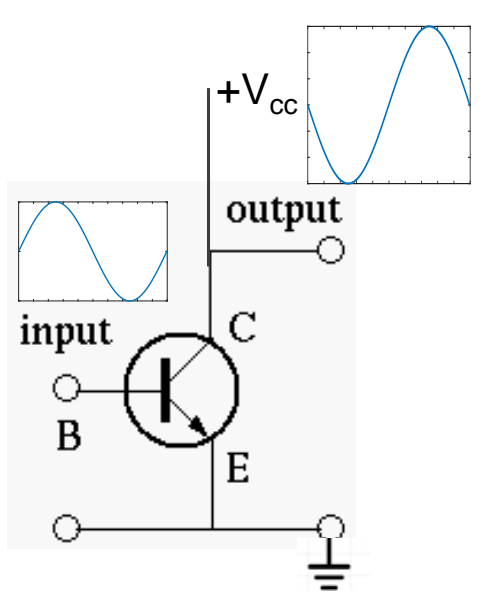

(b)

Fig. 5. Block schematic (a) and basic circuit configuration of a power-amplifying transistor The power-added efficiency is essentially the efficiency of power conversion from mains electricity to microwaves (for unity power supply efficiency).

\subsection{Solid-state versus classical magnetrons}

Solid-state power amplifiers are more advantageous than magnetrons, primarily because of their ability to receive feedback on the dynamic states of the processed food, and control same, using different degrees of freedom like frequency, phase and power amplitude - sometimes, using multiple generators. Atuonwu and Tassou (2017) itemised (with details), the advantages of solid-state over magnetrons to include:

i. Accessibility to feedback and process optimisation algorithms

ii. Possibility of multiple power feeds with independent control

iii. Frequency accuracy and stability

iv. Frequency control

v. Phase control

vi. Power amplitude control

vii. Repeatability and reproducibility of product quality

viii. Reliability and lifetime - 15 years continuous operation as against about 500 hours in magnetron-powered household ovens, and one year of continuous use in commercial and industrial environments (Wesson, 2015).

ix. Size and operational voltages - about 50V or less compared to the 4-20 kV of magnetrons, at least, 10 times lighter for the same wattage

x. Improved compatibility with other electronic circuitry, and the Internet-ofThings in the future (Jerby, 2016).

Frequency, phase and power amplitude control as well as multi-source power delivery have all been individually shown to improve heating uniformity in several studies (Bows, 1999; Antonio and Deam, 2005; Gunasekaran and Yang, 2007; DominguezTortajada et al., 2007; Liao et al., 2016; Bae et al., 2017). When combined effectively in solid-state systems, more significant improvements in uniformity are expected. This also implies improvements in absorption and hence, overall energy efficiency. Moreover, by taking advantage of relevant optimisation algorithms, the system can be 
steered to minimise reflections, and hence attain absorption efficiencies, close to $100 \%$ as shown a simulation study (Korpas et al., 2013).

\subsection{Techno-economic analysis of solid-state versus magnetron options}

Acquisition costs and energy-efficiency issues have in the past, hindered the adoption of solid-state technology in microwave heating applications (Schwarz, et al., 2006). As at 2006, when that article was written, the maximum power output of the cheapest solid-state devices (Laterally Diffused Metal Oxide Silicon LDMOS technology) was about $180 \mathrm{~W}$, with power-added efficiencies of about $46 \%$ at $2450 \mathrm{MHz}$. Other technologies such as Gallium Arsenide (GaAs), Silicon Carbide (SiC) and Gallium Nitride (GaN), in packaged, chip and Monolithic Microwave Integrated Circuit (MMIC) were and are still significantly more expensive and sometimes with lower energy performances. The power outputs and efficiencies have however increased tremendously as shown in Table 7 (a representative summary of currently available solid-state power generators, with power output and drain efficiencies, at least $200 \mathrm{~W}$ and $50 \%$, respectively, operating within the frequency ranges relevant for food processing). As seen in the Table, power levels of $1000 \mathrm{~W}$ are possible (for $896 / 915 \mathrm{MHz}$ operation), and $300 \mathrm{~W}$ (for $2450 \mathrm{MHz}$ operation). Power-added efficiencies of $69 \%$ as also possible at both frequencies. This is comparable to what obtains with magnetrons at $2450 \mathrm{MHz}$, and gradually approaching the $88 \%$ attainable with magnetrons at $896 / 915 \mathrm{MHz}$. When multiplied by the absorption efficiencies (see equations $1,2 \& 3$ ), the electricity-to-heat energy efficiency of solid-state systems is expected to be higher than that of magnetron-based systems, especially at $2450 \mathrm{MHz}$.

For the more cost-effective LDMOS technology, an efficiency (generation) of $61 \%$ at $206 \mathrm{~W}$ is possible at $2450 \mathrm{MHz}$. The current rate of efficiency improvement, which has been valid over the last decade, is about 3\% every 2-3 years (Wesson, 2016). Based on this and other information on typical experimentally-obtained absorption efficiencies for magnetron- and solid-state-based systems, plots are produced (Fig. 6) to compare the different aspects of energy efficiency over a period of years. Two scenarios are considered for $2450 \mathrm{MHz}$ systems:

i. The evolution of solid-state and magnetron system energy efficiencies, from the past decade is used to project into the next 15 years, to predict possible break-even points

ii. Given that a solid-state- and magnetron-based microwave processing system are put into service, this year, energy-efficiency projections are made over the next 15 years, taking into consideration, possible energy performance depreciations that have been observed to occur

To achieve this, the following reasonably valid assumptions are made: 
a. The average absorption efficiency of magnetron-based systems is taken as $65 \%$, based on Wang et al. (2015b), while that of the solid-state system is 88.4\% (Durnan, 2016).

b. The current rate of efficiency improvement (3\% every 2-3 years), which has been valid over the last decade, (Wesson, 2016), is assumed to remain valid over the next 15 years.

c. The generation efficiency of magnetron-based systems drops by about $33 \%$ for every 10 years. This is in fact a conservative estimate, based on Anantheswaran (1999). A linear depreciation is assumed. Actual depreciation depends on the frequency of use, with commercial systems depreciating much faster than household systems. Solid-state systems are assumed to maintain their initial generation efficiencies, throughout service life (Wesson, 2016). 
Table 7. List of currently-available solid-state microwave generators in the frequency ranges relevant to industrial (top part) and domestic (bottom part) food processing operations. Courtesy https://www.rfmw.com/

\begin{tabular}{|c|c|c|c|c|c|c|c|c|c|}
\hline Part number & Manufacturer & Process & $\begin{array}{l}\text { Min. } \\
\text { freq. } \\
(\mathrm{GHz})\end{array}$ & $\begin{array}{l}\text { Max. } \\
\text { freq. } \\
(\mathrm{GHz})\end{array}$ & $\begin{array}{l}\text { Output } \\
\text { power } \\
\text { (W) }\end{array}$ & $\begin{array}{l}\text { Gain } \\
\text { (dB) }\end{array}$ & $\begin{array}{l}\text { Supply } \\
\text { voltage } \\
\text { (V) }\end{array}$ & $\begin{array}{l}\text { Drain } \\
\text { efficiency } \\
\text { (\%) }\end{array}$ & $\begin{array}{l}\text { Power- } \\
\text { added } \\
\text { efficiency } \\
(\%)\end{array}$ \\
\hline MMRF1312GSR5 & NXP & LDMOS & 0.9 & 1.215 & 1000 & 17.3 & 52 & 54.00 & 52.99 \\
\hline MMRF1312HSR5 & NXP & LDMOS & 0.9 & 1.215 & 1000 & 17.3 & 52 & 54.00 & 52.99 \\
\hline MMRF1312HR5 & NXP & LDMOS & 0.9 & 1.215 & 1000 & 17.3 & 52 & 54.00 & 52.99 \\
\hline MRF13750HSR5 & NXP & LDMOS & 0.7 & 1.3 & 750 & 19.3 & 50 & 67.10 & 66.31 \\
\hline MRF13750HR5 & NXP & LDMOS & 0.7 & 1.3 & 750 & 19.5 & 50 & 67.10 & 66.35 \\
\hline PRF13750HR9 & NXP & LDMOS & 0.7 & 1.3 & 750 & 19.5 & 50 & 63.00 & 62.29 \\
\hline BLF988 & Ampleon & LDMOS & 0.5 & 1 & 600 & 20 & 50 & 58.00 & 57.42 \\
\hline BLF988S & Ampleon & LDMOS & 0.5 & 1 & 600 & 20 & 50 & 58.00 & 57.42 \\
\hline BLF0910H6LS500U & Ampleon & LDMOS & 0.9 & 0.93 & 500 & 18 & 50 & 61.00 & 60.03 \\
\hline BLA8H0910LS-500U & Ampleon & LDMOS & 0.9 & 0.93 & 500 & 19 & 50 & 62.50 & 61.71 \\
\hline BLF0910H6L500U & Ampleon & LDMOS & 0.9 & 0.93 & 500 & 18 & 50 & 61.00 & 60.03 \\
\hline BLA8H0910L-500U & Ampleon & LDMOS & 0.9 & 0.93 & 500 & 19.5 & 50 & 62.50 & 61.80 \\
\hline MHT1002GNR3 & NXP & LDMOS & 0.915 & 0.915 & 387 & 20.7 & 48 & 66.90 & 66.33 \\
\hline MHT1002NR3 & NXP & LDMOS & 0.915 & 0.915 & 387 & 20.7 & 48 & 66.90 & 66.33 \\
\hline MRF8VP13350NR3 & NXP & LDMOS & 0.7 & 1.3 & 350 & 20.7 & 50 & 67.50 & 66.93 \\
\hline MRF8VP13350GNR3 & NXP & LDMOS & 0.7 & 1.3 & 350 & 20.7 & 50 & 67.50 & 66.93 \\
\hline T1G2028536-FL & Qorvo & GaN & 0 & 2 & 285 & 18 & 36 & 65.60 & 64.56 \\
\hline T1G2028536-FS & Qorvo & GaN & 0 & 2 & 285 & 18 & 36 & 65.60 & 64.56 \\
\hline BLF6G13L-250P & Ampleon & LDMOS & 0.005 & 1.3 & 250 & 17 & 50 & 56.00 & 54.88 \\
\hline BLF6G13LS-250P & Ampleon & LDMOS & 0.005 & 1.3 & 250 & 17 & 50 & 56.00 & 54.88 \\
\hline A2V09H300-04NR3 & NXP & LDMOS & 0.72 & 0.96 & 223 & 19.7 & 48 & 55.90 & 55.30 \\
\hline BLF647PSJ & Ampleon & LDMOS & 0 & 1.4 & 200 & 18 & 32 & 70.00 & 68.89 \\
\hline BLF647PS & Ampleon & LDMOS & 0 & 1.4 & 200 & 18 & 32 & 70.00 & 68.89 \\
\hline BLF647P & Ampleon & LDMOS & 0 & 1.4 & 200 & 18 & 32 & 70.00 & 68.89 \\
\hline BLC2425M8LS300P & Ampleon & LDMOS & 2.4 & 2.5 & 300 & 17 & 32 & 58.00 & 56.84 \\
\hline MRF24300NR3 & NXP & LDMOS & 2.4 & 2.5 & 300 & 13.1 & 32 & 60.50 & 57.54 \\
\hline MHT1004NR3 & NXP & LDMOS & 2.45 & 2.45 & 280 & 15.2 & 32 & 57.90 & 56.15 \\
\hline MHT1004GNR3 & NXP & LDMOS & 2.45 & 2.45 & 280 & 15.2 & 32 & 57.90 & 56.15 \\
\hline MHT1003NR3 & NXP & LDMOS & 2.45 & 2.45 & 263 & 15.9 & 32 & 59.00 & 57.48 \\
\hline BLF2425M7LS250P & Ampleon & LDMOS & 2.4 & 2.5 & 250 & 13 & 28 & 55.00 & 52.24 \\
\hline BLF2425M7L250P & Ampleon & LDMOS & 2.4 & 2.5 & 250 & 15 & 28 & 51.00 & 49.39 \\
\hline MRF7S24250NR3 & NXP & LDMOS & 2.4 & 2.5 & 250 & 14.7 & 32 & 54.80 & 52.94 \\
\hline BLF2425M7L250P & Ampleon & LDMOS & 2.4 & 2.5 & 250 & 13 & 28 & 55.00 & 52.24 \\
\hline BLC2425M9LS250Z & Ampleon & LDMOS & 2.4 & 2.5 & 250 & 18.5 & 32 & 61.00 & 60.14 \\
\hline MHE1003NR3 & NXP & LDMOS & 2.45 & 2.45 & 206 & 14.1 & 26 & 63.50 & 61.03 \\
\hline T1G4020036-FL & Qorvo & GaN & 0 & 3.5 & 200 & 18.1 & 50 & 68.66 & 67.60 \\
\hline T1G4020036-FS & Qorvo & GaN & 0 & 3.5 & 200 & 18.1 & 50 & 69.68 & 68.60 \\
\hline
\end{tabular}

The results of these projections are as presented in Fig. 6(a) - (d). From Fig. 6(a), it is seen that solid-state microwave generation efficiencies, have been steadily increasing since 2006, up till date. Although still less than that of the magnetron, a break-even point is expected at about 2024, all things being equal. The lower generation efficiency is however more than compensated for, by the significant increase in absorption efficiency, offered by the solid-state system. Consequently, the evolutionary overall energy efficiency as shown in Fig. 6(b), is already higher than that of a magnetron-based system, having broken even in about 2009. Assuming a solid-state- and 

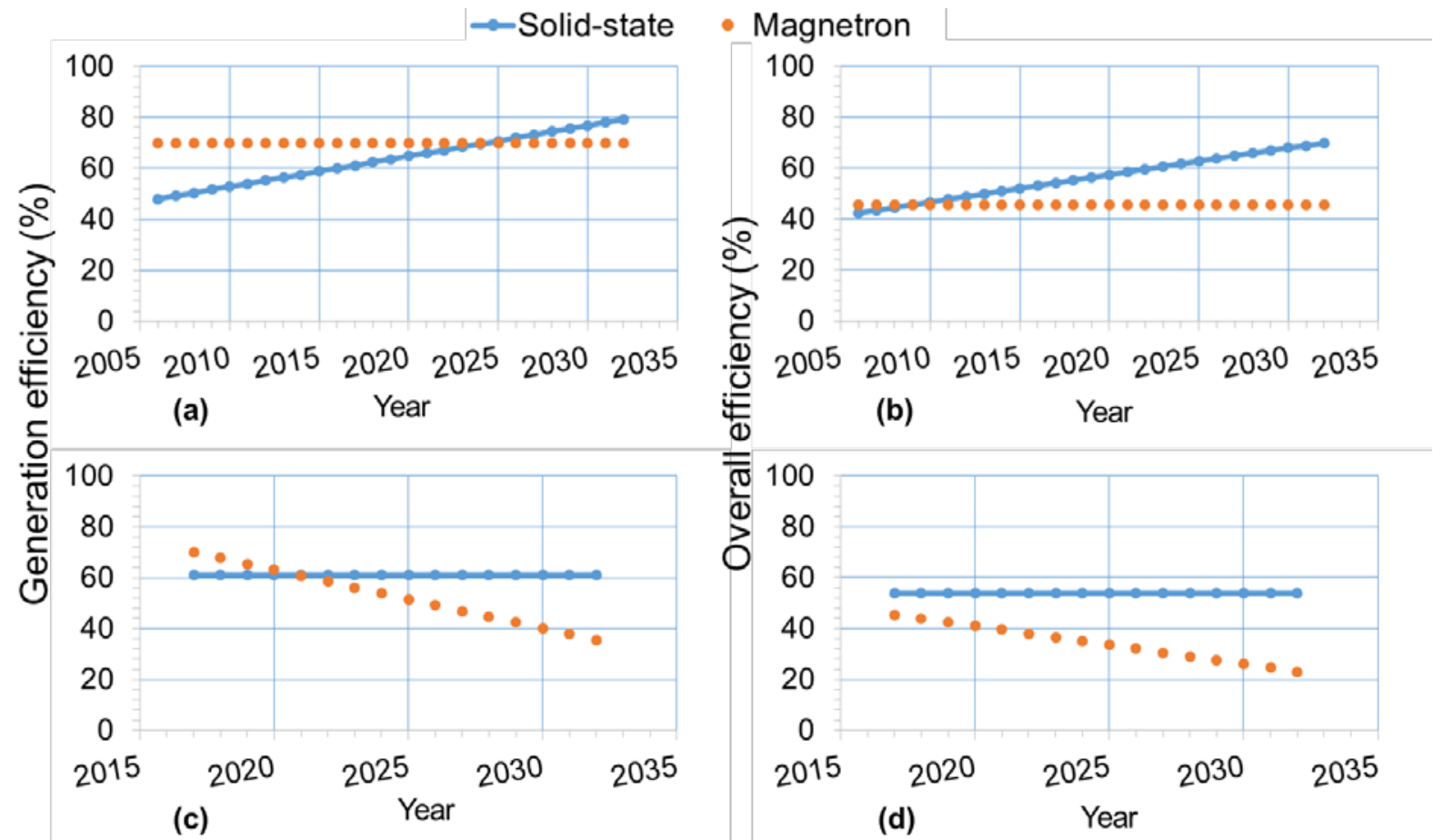

Fig. 6. Energy efficiencies of solid-state- and magnetron-based systems under different scenarios

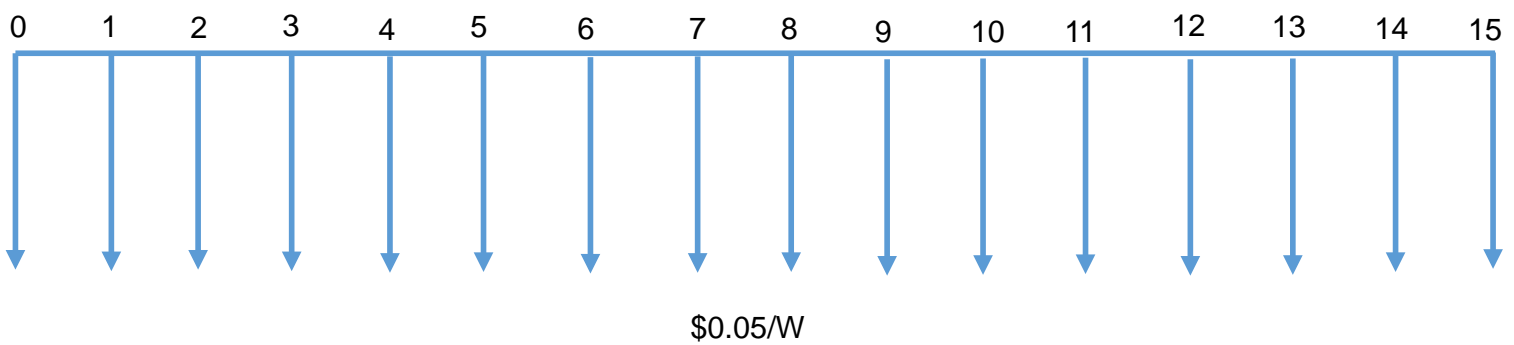

(a)

$\$ 0.05 / \mathrm{W}$

\begin{tabular}{llllllllllllllll}
0 & 1 & 2 & 3 & 4 & 5 & 6 & 7 & 8 & 9 & 10 & 11 & 12 & 13 & 14 & 15 \\
\hline
\end{tabular}

(b)

$\$ 0.25 / \mathrm{W}$

Fig. 7. Cash flow diagrams for magnetron (a) and solid-state (b) - based systems

magnetron-based system are put into service today, by 2021 (as seen in Fig. 6(c)), the generation efficiency of the magnetron system is expected to start dropping below that of the solid-state system, owing to the linear depreciation of output with time. The overall efficiency of the solid-state system is however already higher than that of the 
magnetron-based system, owing to the vastly improved absorption efficiency brought about by the solid-state system (Fig. 6(d)). The difference is expected to increase as the years go by. Hence, even for initially-higher acquisition costs, it is possible to break even economically, within a short payback period.

In terms of acquisition costs, solid-state generators are still more expensive. For the cheapest technology LDMOS, $\$ 0.25 / \mathrm{W}$, is now achievable in some cases as against $>\$ 1.00 / \mathrm{W}$ in the past (compare Fitzpatrick, 2013 and Schwartz, et al., 2006). Magnetrons cost $\$ 0.01 \mathrm{~W}$ to $\$ 0.1 \mathrm{~W}$ (Edgar and Osepchuk, 2001). It is however noteworthy that magnetron-based commercial/industrial systems have a limited lifetime of 1 year. We estimate the overall economic impact of this situation using the "Net Present Value" metrics. Negative cash flows are assumed to be made at the end of every year, for the magnetron-based system, to cater for replacement costs (a value of $\$ 0.05 \mathrm{~W}$ is assumed, each year); while for the solid-state system, there are no replacements over 15 years. The cash flow diagrams for both cases are presented in Fig. $7(a) \&(b)$. The net present value is then calculated using the relation

$$
N P V=\sum_{t=0}^{N} \frac{R_{t}}{(1+i)^{t}}
$$

where $R_{t}$ is the net cash flow at year $t$ (see Fig. $7(a) \&(b)$ ), $N=15$ the total number of years under consideration, and $i=10 \%$, the discount rate. The results as shown in Fig. 8 indicate that after about 5 years, the net magnetron cash flow becomes more negative than the solid-state case. This is equivalent to the payback period, neglecting other advantages like energy savings and quality improvements, which usually minimise product wastage and recycle costs. It can therefore be inferred that the use of solid-state amplifiers as replacements to magnetrons is promising, not only from an energy and overall technical perspective, but also in terms of economics.

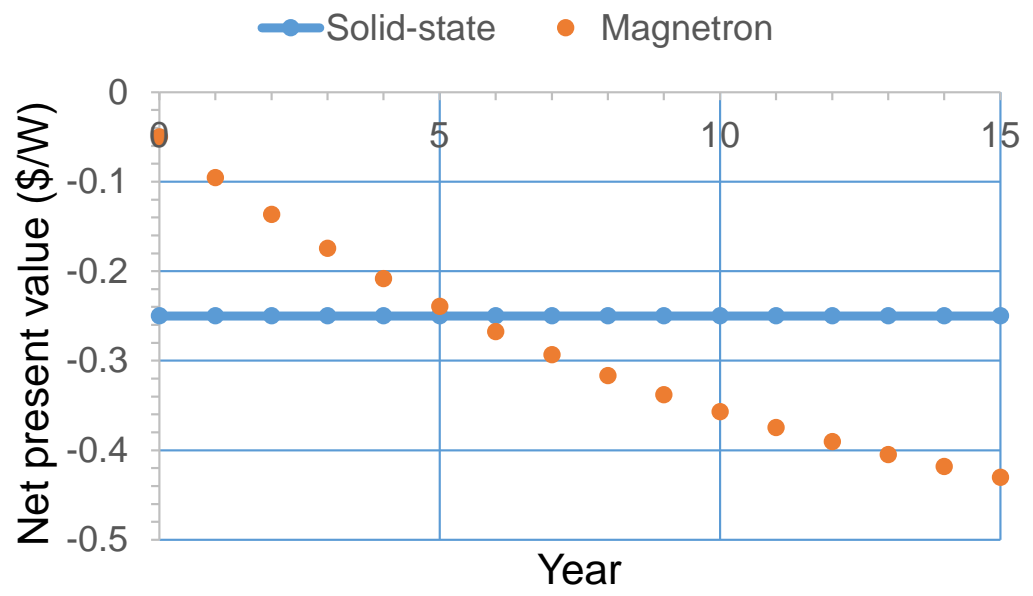

Fig. 8. Net present value results for magnetron (a) and solid-state (b) - based systems 


\section{Conclusions}

Key energy issues associated with the microwave processing of foods have been reviewed and critically accessed, from which the following can be inferred:

- With the exception of microwave and microwave-assisted food drying operations, not much is available in the literature on studies dedicated specifically to energy consumption analysis of microwave food processes.

- Of the drying-related studies, majority are in the domestic scale, with perhaps only one study in the $915 \mathrm{MHz}$ frequency range

- Microwaves are capable of improving energy performances, compared to conventional drying, provided the microwave power level is properly chosen to match the moisture loading and overall characteristics of the processed food material, minimising reflections. In combination operations, additional degrees of freedom are introduced which improve energy optimisation possibilities.

- The energy performance indices "thermal efficiency" and "drying efficiency", as currently used in the microwave drying literature have significantly different definitions from the same terms as used in the conventional drying literature. A unification of definitions is suggested to facilitate comparison.

- From an energy standards and regulations perspective, it is important to implement energy labelling schemes for microwave heaters in the European Union, which is currently not the case. Such labelling, should take advantage of the recently-implemented European Standards metric: the "energy consumption of a cooking cycle to reach a temperature increase of $50 \mathrm{~K}$ ", as this is more indicative of actual food processing conditions than the standard $1000 \mathrm{~g}, 10 \mathrm{~K}$ temperature rise power calibration measurements.

- Factors affecting energy-efficiency are categorised into source, load and source-load matching factors. This highlights the need for highly-flexible and controllable power sources capable of receiving real-time feedback on load properties, and effecting rapid control actions to minimise reflections, heating non-uniformities and other imperfections that lead to energy losses.

- Solid-state amplifiers are promising replacements to magnetrons as microwave power sources not only from an energy and overall technical perspective, but also in terms of economics; going by the results of a techno-economic analysis

\section{Acknowledgements}

The work presented in this paper received funding from the Research Councils UK (RCUK) for the establishment of the RCUK Centre for Sustainable Energy Use in Food Chains (CSEF) through EPSRC grant No: EP/K011820/1. The authors acknowledge the financial support from the Research Councils UK Energy Programme and support received from CSEF. All data used are given in the paper but the corresponding author can be contacted for additional information or data if required. 


\section{References}

Aghilinategh, N., Rafiee, S., Hosseinpur, S., Omid, M., Mohtasebi, S.S. (2015). Optimization of intermittent microwave-convective drying using response surface methodology. Food Science and Nutrition 3(4), 331-341.

Alibas, I. (2007a). Energy consumption and Colour characteristics of nettle leaves during microwave, vacuum and convective drying. Biosystems Engineering 96(4), 495-502.

Alibas, I. (2007b). Microwave, air and combined microwave-air-drying parameters of pumpkin slices. Lebensmittel-Wissenschaft \& Technologie 40, 1445-1451.

Anantheswaran, S. (1999). Microwave ovens can be zapped by age factors. Personal Communications

Antonio, C., Deam, R.T. (2005). Comparison of linear and non-linear sweep rate regimes in variable frequency microwave technique for uniform heating in materials processing. Journal of Materials Processing Technology 169(2), 234-241.

Atuonwu, J.C., Straten, G. van., Deventer, H.C. van., Boxtel, A.J.B. van. (2012). On the controllability and energy sensitivity of heat-integrated desiccant adsorption dryers. Chemical Engineering Science 80, 134-147.

Atuonwu, J.C., Tassou, S.A. (2017). Quality assurance in microwave food processing: a review of developments and the enabling potentials of solid-state power delivery. "Unpublished".

Bae, S., Jeong, M., Kim, J., Lee, W. (2017). A continuous power-controlled microwave belt dryer improving heating uniformity. IEEE Microwave and wireless components letters 27(5), 527-529.

Bahl, I. (2009). Fundamentals of RF and microwave transistor amplifiers. John Wiley and Sons Inc., NJ.

Bermúdez, J.M., Beneroso, D., Rey-Raap, N., Arenillas A., Menéndez, J.A. (2015). Energy consumption estimation in the scaling-up of microwave heating processes. Chemical Engineering and Processing: Process Intensification.

Bows, J.R. (1999). Variable frequency microwave heating of food. Journal of Microwave Power and Electromagnetic Energy, 34(4), 227-238.

BS EN 60705 (2015). Household microwave ovens - Methods for measuring performance. BSI Standards Publication, London.

Chandrasekaran, S., Ramanathan, S., Basak, T. (2013). Microwave food processing-a review. Food Research International 52, 243-261.

Darvishi, H., Zarein, M., Minaei, S., Khafajeh, H. (2014). Exergy and energy analysis, drying kinetics and mathematical modelling of white mulberry drying process. International Journal of Food Engineering doi 10.1515/ijfe-2013-0065.

Darvishi, H. (2012). Energy consumption and mathematical modelling of microwave drying of potato slices. Agricultural Engineering International: CIGR Journal 14(1), 94-102.

Devine, W.G., Leadbeater, N.E. (2011). Probing the energy efficiency of microwave heating and continuous-flow conventional heating as tools for organic chemistries. ARKIVOC 127-143.

Ding, S., You, J., An, K., Li, Y., Wang, Z. (2012). Effective diffusivities and energy consumption of daylily in microwave drying. International Journal of Food Science and Technology 47, 2648-2654.

Dominguez-Tortajada, E., Plaza-Gonzalez, P., Diaz-Morcillo, A., Balbastre, J.V. (2007). Optimisation of electric field uniformity in microwave heating systems by means of multi-feeding and genetic algorithms. International Journal of Materials and Product Technology 29(1-4), 149-162.

Durnan, G. (2016). Solid-State Heating with Advanced RF-Power Solutions. AMPERE Newsletter 89, 13-17.

EC (2017). Cooking appliances. The European Commission, Accessed 11/10/2017 from https://ec.europa.eu/energy/en/topics/energy-efficiency/energy-efficient-products/cookingappliances

Edgar, R., Osepchuk, J.M. (2001). Consumer, commercial and industrial microwave ovens, In Datta, A.K., Anantheswaran, R. (Eds.), Handbook of Microwave technology and Food Application, pp. 215-277, Marcel Dekker, New York.

Ekezie, F.C., Sun, D., Han, Z., Cheng, J. (2017). Microwave-assisted food processing technologies for enhancing product quality and process efficiency: A review of recent developments. Trends in Food Science and Technology 67, 58-69.

Euromonitor International (2017). Microwaves in the United Kingdom. Accessed 12/09/2017 from http://www.euromonitor.com/microwaves-in-the-united-kingdom/report 
Fang, S., Wang, Z., Hu, X., Chen, F., Zhao, G., Liao, X., Wu, J. Zhang, Y. (2009). Energy requirement and quality aspects of Chinese jujube (Zizyphus jujuba miller) in hot air drying followed by microwave drying. Journal of Food Process Engineering 34, 491-510.

Fitzpatrick, D. (2013). Recent developments in high power solid-state power amplifiers: a European perspective. Powerful Microwave, Automated RF and Microwave Measurement Society Conference, November 2013, Wyboston, UK.

Gentry, T.S., Roberts, J.S. (2004). Formation kinetics and application of 5-hydroxymethylfurfural as a time-temperature indicator of lethality for continuous pasteurization of apple cider. Innovative Food Science and Emerging Technologies 5, 327-333.

Gentry, T.S., Roberts, J.S. (2005). Design and evaluation of a continuous microwave pasteurisation system for apple cider. Lebensmittel-Wissenschaft \& Technologie 38, 227-236.

Göllei, A., Vass, A., Magyar, A., Pallai, E. (2009). Apparatus and method for investigation of energy consumption of microwave assisted drying systems. Review of Scientific Instruments 80, 104706, doi: 10.1063/1.3250870.

Gunasekaran, S., Yang, H. (2007). Effect of experimental parameters on temperature distribution during continuous and pulsed microwave heating. Journal of Food Engineering 78, 1452-1456.

Guo, Q., Sun, D., Cheng, J., Han, Z. (2017). Microwave processing techniques and their recent applications in the food industry. Trends in Food Science \& Technology 67, 236-247.

Hazervazifeh, A., Moghaddam, P.A., Nikbahkt, A.M. (2017). Microwave dehydration of apple fruit: Investigation of drying efficiency and energy costs. Journal of Food Process Engineering 40:e12463. https://doi.org/10.1111/jfpe.12463.

HEECS (2010). High efficiency electronic cooking systems. Accessed 12/09/2017 from http://www.eniac.eu/web/downloads/projectprofiles/call3 heecs.pdf

HEECS (2011). High efficiency electronic cooking systems. Review of existing standards: gaps and constraints. Accessed 11/10/2017 from http://www.heecs.eu/wordpress/wpcontent/uploads/2012/03/HEECS-D2.2-M6.pdf

Holtz, E., Ahrné, L., Rittenauer, M., Rasmuson, A. (2010). Influence of dielectric and sorption properties on drying behaviour and energy efficiency during microwave convective drying of selected food and non-food inorganic materials. Journal of Food Engineering 97, 144-153.

Hoogenboom, R., Wilms, T.F., Erdmenger, T., Schubert, U.S. (2009). Microwave-assisted chemistry: a closer look at heating efficiency. Australian Journal of Chemistry 62, 236-243.

IEC 60705 (1999). Household microwave ovens - methods for measuring performance. International Electrotechnical Commission, IEC Publication, Geneva.

Jafari, H., Kalantari, D., Azadbakht, M. (2017). Semi-industrial continuous band microwave dryer for energy and exergy analyses, mathematical modelling of paddy drying and its qualitative study. Energy 138, 1016-1029.

Jerby, E. (2016). Editorial. AMPERE Newsletter. Trends in RF and microwave heating. Special Issue on Solid-State Microwave Heating 89, 2.

Jiang, N., Liu, C., Li, D., Zhang, Z., Liu, C., Wang, D., Niu, L., Zhang, M. (2017). Evaluation of freeze drying combined with microwave vacuum drying for functional okra snacks: Antioxidant properties, sensory quality, and energy consumption. LWT-Food Science and Technology 82, 216-226.

Jindarat, W., Rattanadecho, P., Vongpradubchai, S., Pianroj, Y. (2011). Analysis of energy consumption in drying process of non-hygroscopic porous packed bed using a combined multifeed microwave-convective air and continuous belt system (CMCB). Drying Technology, 29(8), 926-938.

Jindarat, W., Sungsoontorn, S., Rattanadecho, P. (2013). Analysis of energy consumption in drying process of biomaterials using a combined unsymmetrical double-feed microwave and vacuum system (CUMV)-case study: tea leaves. Drying Technology 31, 1138-1147.

Khoshtaghaza, M.H., Darvishi, H., Minaei, S. (2015). Effects of microwave - fluidized bed drying on quality, energy consumption and drying kinetics of soybean kernels. Journal of Food Science and Technology 52(8), 4749-4760.

Kim S, Eriksson E, Lee K (1996): Screening LCA of a microwave oven. Proceedings of SETAC Case Study Symposium, December, Brussels, Belgium, 35. 
Korpas, P., Wieckowski, A., Krysicki, M., Celuch, M. (2013). Application study of new solid-state highpower microwave sources for efficiency improvement of commercial domestic ovens. Proceedings, 47th Microwave Power Symposium, Rhode Island.

Kudra, T. (2004). Energy aspects in drying. Drying Technology 22(5), 917-932.

Kurják, Z., Barhács, A., Beke, J. (2012). Energetic analysis of drying biological materials with high moisture content by using microwave energy. Drying Technology 30(3), 312-319.

Lakshmi, S., Chakkaravarthi, A., Subramanian, R., Singh, V. (2007). Energy consumption in microwave cooking of rice and its comparison with other domestic appliances. Journal of Food Engineering 78, 715-722.

Lechtanska, J. M., Szadzinska, S., Kowalski, S.J. (2015). Microwave- and infrared-assisted convective drying of green pepper: quality and energy considerations. Chemical Engineering and Processing: Process Intensification 98, 155-164.

Liao, Y., Lan, J., Zhang, C., Hong, T., Yang, Y., Huang, K., Zhu, H. (2016). A phase-shifting method for improving the heating uniformity of microwave processing materials. Materials 9, 309; doi:10.3390/ma9050309.

Lule, F., Koyuncu, T. (2017). Convective and microwave drying characteristics, energy requirement and color retention of dehydrated nettle leaves (Urtica diocia L.). Legumes Research 40(4), 649654.

Menshutina, N.V., Gordienko, M. G., Voynovskiy, A. A., Kudra, T. (2004). Dynamic analysis of drying energy consumption. Drying Technology 22(10), $2281-2290$.

Metaxas, A.C. (1991). Microwave heating. Power Engineering Journal 5(5), 237-247.

Microwave Technologies Association (2017). Fact sheets, accessed 13/09/2017 from http://www.microwaveassociation.org.uk/factsheets/facts.php

MOE (2015). Top Runner Program, Developing the world's best energy-efficient appliance and more. Ministry of Economy, Trade and Industry. Agency for Natural Resource and Energy pp. 77.

Morschhäuser, R., Krull, M., Kayser, C., Boberski, C., Bierbaum, R., Püschner, P.A., Glasnov, T.N., Kappe, C.O. (2012). Microwave-assisted continuous flow synthesis on industrial scale. Green Process Synthesis 1, 281-290.

Moseley, J.D., Kappe, C.O. (2011). A critical assessment of the greenness and energy efficiency of microwave-assisted organic synthesis, Green Chemistry 13, 794-806.

Motevali, A., Minaei, S., Khoshtaghaza, M.H., Amirnejat, H. (2011). Comparison of energy consumption and specific energy requirements of different methods for drying mushroom slices. Energy 36, 6433-6441.

Motevali, A., Minaei, S., Banakar, A., Ghobadian B., Darvishi, H. (2016). Energy analyses and drying kinetics of chamomile leaves in microwave-convective dryer. Journal of the Saudi Society of Agricultural Sciences 15, 179-187.

Mousa, N., Farid, M. (2002). Microwave vacuum drying of banana slices. Drying Technology 20(10), 2055-2066.

Olatunde, G.A., Atungulu, G.G., Smith, D.L. (2017). One-pass drying of rough rice with an industrial $915 \mathrm{MHz}$ microwave dryer: Quality and energy use consideration. Biosystems Engineering 155, 33-43.

ONSUK (2017). Percentage of households with microwave ovens in the United Kingdom (UK) from 1994 to 2014. Office for National Statistics, UK. Accessed 14/09/2017 from https://www.statista.com/statistics/289155/household-microwave-penetration-in-the-uk/

Osepchuk, J. (2002). Microwave power applications. IEEE Transactions on Microwave Theory and Techniques, 50(3), 975-985.

Schwartz, E., Anaton, A., Jerby, E. (2006). Transistor-based microwave heaters. Proceedings, 40th Annual Microwave Symposium, 246-249.

Soysal, Y., Oztekin, S., Eren, O. (2006). Microwave drying of parsley: modelling, kinetics, and energy aspects. Biosystems Engineering 93(4), 403-413.

Swain, M.J., Ferron, S., Coelho, A.I.P., Swain, M.V.L. (2006). Effect of continuous (intermittent) use on the power output of domestic microwave ovens. International Journal of Food Science and Technology 41, 652-656.

Vadivambal, R., Jayas, D.S. (2010). Non-uniform temperature distribution during microwave heating of foods - a review. Food and Bioprocess Technology 3, 161-171. 
Varith, J., Dijkanarukkul, P., Achariyaviriya, A., Achariyaviriya, S. (2007). Combined microwave-hot air drying of peeled longan. Journal of Food Engineering 81, 459-468.

Wang, Z., Fang, S., Hu, X. (2009). Effective diffusivities and energy consumption of whole fruit Chinese jujube (Zizyphus jujuba Miller) in microwave drying. Drying Technology, 27(10), 1097-1104.

Wang, H., Guo, K., Li, P., Lin, Y. (2015a). Microwave drying kinetics and energy consumption analysis of extruded cotton stalks. Transactions of the Chinese Society of Agricultural Engineering 31(9), 294-301.

Wang, W., Zhao, C., Sun, J., Wang, X., Zhao, X., Mao, Y., Li, X., Song, Z. (2015). Quantitative measurement of energy utilization efficiency and study of influence factors in typical microwave heating process. Energy 87, 678-685.

Werner, K. (2015). RF energy systems: realizing new applications. Microwave Journal 1-8, December 11.

Wesson, R. (2015). RF solid-state cooking white paper. Ampleon, pp. 1-13, www.ampleon.com

Wesson, R. (2016). Solid-state microwave cooking. AMPERE Newsletter 89, 27-32.

Yongsawatdigul, J., Gunasekaran, S. (1996). Microwave-vacuum drying of cranberries: part i. energy use and efficiency. Journal of Food Processing and Preservation 20(2), 121-143.

Zarein, A., Samadi, S.H., Ghobadian, B. (2015). Investigation of microwave dryer effect on energy efficiency during drying of apple slices. Journal of the Saudi Society of Agricultural Sciences 14, 41-47. 